\title{
Structural basis for repurposing a 100-years-old drug suramin for treating COVID-19
}

H. Eric Xu ( $\nabla$ eric.xu@simm.ac.cn )

Shanghai Institute of Materia Medica (SIMM) https://orcid.org/0000-0002-6829-8144

Wanchao Yin

Shanghai Institute of Materia Medica (SIMM)

Xiaodong Luan

Tsinghua University,

\section{Zhihai Li}

Shanghai Institute of Materia Medica https://orcid.org/0000-0002-2816-6243

\section{Leike Zhang}

Chinese Academy of Sciences https://orcid.org/0000-0002-2593-2571

\section{Ziwei Zhou}

Shanghai Institute of Materia Medica (SIMM)

\section{Minqi Gao}

WuxiBiortus Biosciences Co. Ltd.

\section{Xiaoxi Wang}

Shanghai Institute of Materia Medica (SIMM)

\section{Zhou Fulai}

Shanghai Institute of Materia Medica (SIMM)

\section{Jingjing Shi}

Shanghai Institute of Materia Medica (SIMM)

\section{Erli You}

Shanghai Institute of Materia Medica (SIMM)

\section{Mingliang Liu}

Shanghai Institute of Materia Medica (SIMM)

\section{Qingxia Wang}

Shanghai Institute of Materia Medica (SIMM)

\section{Qingxing Wang}

Wuhan Institute of Virology

\section{Yi Jiang}

Shanghai Institute of Materia Medica https://orcid.org/0000-0002-0723-1413

\section{Hualiang Jiang}

Shanghai Institute of Materia Medica https:// orcid.org/0000-0003-0656-6315

\section{Gengfu Xiao}


Wuhan Institute of Virology, Chinese Academy of Sciences

\section{Xuekui Yu}

Shanghai Institute of Materia Medica (SIMM)

\section{Shuyang Zhang}

Peking Union Medical College Hospital

\section{Article}

Keywords: suramin, COVID-19, SARS-CoV-2

Posted Date: November 5th, 2020

DOI: https://doi.org/10.21203/rs.3.rs-99513/v1

License: (c) (i) This work is licensed under a Creative Commons Attribution 4.0 International License. Read Full License

Version of Record: A version of this preprint was published at Nature Structural \& Molecular Biology on March 5th, 2021. See the published version at https://doi.org/10.1038/s41594-021-00570-0. 


\section{Structural basis for repurposing a 100-years-old drug suramin for treating COVID-19}

WanchaoYin ${ }^{1,9}$, Xiaodong Luan²,3,4,9, Zhihai Li ${ }^{1,5,9}$, Leike Zhang ${ }^{6,9}$, Ziwei Zhou ${ }^{1,5,9}$, Minqi Gao ${ }^{8}$, Xiaoxi Wang ${ }^{1}$, Fulai Zhou ${ }^{1}$, Jingjing Shi ${ }^{1}$, Erli You ${ }^{1}$, Mingliang Liu ${ }^{1}$, Qingxia Wang ${ }^{1,5}$, Qingxing Wang $^{6}$, Yi Jiang ${ }^{1,7}$, Hualiang Jiang ${ }^{1,7}$, Gengfu Xiao ${ }^{6^{*}}$, Xuekui $\mathrm{Yu}^{1,5,7^{\star}}$, Shuyang Zhang ${ }^{3,4,2^{*}}$, H. Eric $\mathrm{Xu}^{1,7^{*}}$

${ }^{1}$ The CAS Key Laboratory of Receptor Research, Shanghai Institute of Materia Medica, Chinese Academy of Sciences, Shanghai 201203, China

${ }^{2}$ School of Medicine, Tsinghua University, Haidian District, Beijing, China

${ }^{3}$ Department of Cardiology, Peking Union Medical College Hospital, Peking Union Medical

College and Chinese Academy of Medical Sciences, Beijing, China

${ }^{4}$ Tsinghua-Peking Center for Life Sciences, Tsinghua University, Beijing, China

${ }^{5}$ Cryo-Electron Microscopy Research Center, Shanghai Institute of Materia Medica, Chinese

Academy of Sciences, Shanghai 201203, China.

${ }^{6}$ State Key Laboratory of Virology, Wuhan Institute of Virology, Center for Biosafety Mega-

Science, Chinese Academy of Sciences, Wuhan, Hubei, 430071, P. R. China

${ }^{7}$ University of Chinese Academy of Sciences, Beijing 100049, China.

${ }^{8}$ WuxiBiortus Biosciences Co. Ltd., 6 Dongsheng West Road, Jiangyin 214437, China

${ }^{9}$ These authors contributed equally to this work

${ }^{*}$ Correspondence and requests for materials should be addressed to H. Eric Xu, eric.xu@simm.ac.cn; or Shuyang Zhang (shuyangzhang103@nrdrs.org ); or Xuekui Yu (xkyu@simm.ac.cn ); or Gengfu Xiao (xiaogf@wh.iov.cn )

One Sentence Summary: Discovery and mechanism of suramin as potent SARS-CoV-2 RNA polymerase inhibitor and its repurposing for treating COVID-19. 


\section{SUMMARY}

The COVID-19 pandemic by non-stop infections of SARS-CoV-2 has continued to ravage many countries worldwide. Here we report the discovery of suramin, a 100-year-old drug, as a potent inhibitor of the SARS-CoV-2 RNA dependent RNA polymerase (RdRp)

through blocking the binding of RNA to the enzyme. In biochemical assays, suramin and its derivatives are at least $\mathbf{2 0}$-fold more potent than remdesivir, the currently approved nucleotide drug for COVID-19. The 2.6 A cryo-EM structure of the viral RdRp bound to suramin reveals two binding sites of suramin, with one site directly blocking the binding of the RNA template strand and the other site clash with the RNA primer strand near the RdRp catalytic active site, therefore inhibiting the viral RNA replication. Furthermore, suramin potently inhibits SARS-CoV-2 duplication in Vero E6 cells. These results provide a structural mechanism for the first non-nucleotide inhibitor of the SARS-CoV-2 RdRp and a rationale for repurposing suramin for treating COVID-19. 


\section{INTRODUCTION}

Severe acute respiratory syndrome coronavirus 2 (SARS-CoV-2) has caused a global pandemic of coronavirus disease 2019 (COVID-19), with over 44.05 million infections and 1.16 million death as reported on October 28 of $2020^{1,2}$. SARS-CoV-2 is a positive-sense, single-stranded RNA virus. In addition to SARS-CoV-2, several related beta-coronaviruses, including SARSCoV and Middle East respiratory syndrome coronavirus (MERS-CoV), are highly pathogenic, which infections can lead to severe acute respiratory syndrome exacerbated by loss of lung function and death. Compared to SARS-CoV and MERS-CoV, SARS-CoV-2 appears to have much higher capacity of human to human infections, which result in a rapidly growing pandemic ${ }^{3}$. Finding an effective treatment for SARS-COV-2 through drug repurposing is an urgent but unmet medical need.

Suramin (Fig. 1a) is a century-old drug that has been used to treat African sleeping sickness and river blindness ${ }^{4,5}$. Suramin has also been shown to be effective in inhibiting the replication of a wide spectrum of viruses, including enteroviruses ${ }^{6}$, Zika virus $^{7}$, Chikungunya $^{8}$ and Ebola viruses $^{9}$. The viral inhibition mechanism of suramin appears to be very diverse, including inhibition of viral attachment, entry, and release from host cells in part through interactions with viral capsid proteins ${ }^{10}$. Recently, suramin has been shown to inhibit SARS-CoV-2 infection in cell culture, which was proposed to act through preventing entry of the virus ${ }^{11}$. Here we report that suramin is a potent inhibitor of the SARS-CoV-2 RNA-dependent RNA polymerase (RdRp), an essential enzyme for the life cycle of virus. The potency of suramin is at least 20 -fold more potent than remdesivir, the current FDA approved nucleotide drug for COVID-19. Cryo-EM structure reveals that suramin binds to the RdRp active site, blocking the binding of both RNA template and primer strands. These results provide a rationale for repurposing suramin for COVID-19 and a structural template to design next generation drugs of suramin derivatives. 


\section{RESULTS and DISCUSSION}

\section{Inhibition of RdRp by suramin and the potential anti-SARS-CoV-2 effect of suramin}

The core RNA polymerase of SARS-CoV-2 is composed of non-structural protein nsp12 with two accessary subunits nsp7 and nsp8 ${ }^{12,13}$. Incubation of the purified nsp12-7-8 complex (Extended Data Fig.1a-1b) with a 30-base template and 20-base primer (poly-A in Fig. 1b) allowed the primer extension to the same length as the template in the presence of saturated concentrations of UTP as illustrated in a gel-based assay (lane 1 in Fig. 1c). Addition of 8-32 $\mu \mathrm{M}$ suramin nearly abolished the elongation of the primer strand while it required $100-1000 \mu \mathrm{M}$ of remdesivir in its triphosphate form (RDV-TP) to achieve the same degree of inhibition under the same condition ${ }^{14}$. Interestingly, addition of $100 \mu \mathrm{M}$ of higher concentrations of suramin completely blocked the formation of RdRp-RNA complex, while it required more than $5 \mathrm{mM}$ of RDV-TP to inhibit the binding of RdRp to RNA (Fig. 1d and Extended Data Fig.1c). Solution based assays of the RdRp inhibition determined that half maximal inhibition concentration (IC $\left.{ }_{50}\right)$ of suramin is $0.26 \mu \mathrm{M}$ (Fig. 1e), and the $\mathrm{IC}_{50}$ for RDV-TP is $6.21 \mu \mathrm{M}$ under identical assay conditions (Extended Data Fig.1d), suggesting that suramin is at least 20 -fold more potent than RDV-TP. Cell-based experiments indicated that suramin was able to inhibit SARS-CoV-2 duplication in Vero E6 cells with a half maximal effective concentration (EC $\left.\mathrm{E}_{50}\right)$ of $\sim 2.9 \mu \mathrm{M}$, which is about the same range as remdesivir in the same assay (Fig. $1 \mathrm{f}$ and Extended Data Fig.1e) ${ }^{15}$. The apparent weaker inhibition of suramin in cell-based assays than in enzyme inhibition assays may be due to the highly negative charges of suramin that may prevent its efficient uptake by the host cells. In addition, the $\mathrm{CC}_{50}$ (concentrations of drug required to reduce cell viability by $50 \%$ ) of suramin is over $1000 \mu \mathrm{M}$, indicating its relatively low cytotoxicity, which is much safer than remdesivir (Fig. 1f and Extended Data Fig.1e).

\section{The structure of the RdRp-suramin complex}

For the cryo-EM studies, we incubated the SARS-CoV-2 RdRp complex with 10-fold molar excess of suramin (see methods). The structure was determined at a nominal resolution of 2.57 $\AA$ with 95,845 particles from over 8 million original particles auto-picked from 11,846 micrographs (Extended Data Fig. 2 and Extended Data Table 1). Because of the relatively high resolution of the structure, the EM map reveal the clear density for all key components of the RdRp-suramin complex, including one nsp12 (residues S6-C22, V31-I106, M110-L895, and N911-T929), one nsp7 (residues K2-G64), two nsp8 (residues D78-A191 for nsp8-1, and residues T84-A191 for nsp8-2, respectively), and two suramin molecules (Fig. 2a and Extended Data Fig. 3). 
The overall structure of the RdRp-suramin complex is very similar to the apo RdRp complex, with an RMSD of $0.465 \AA$ for all Ca atoms between the two structures (Fig. $2 b$ and Extended Data Fig.4). Nsp12 adopts the same right-hand palm-fingers configuration, where its catalytic active site is composed of seven highly conserved motifs A-G (Fig. 3a). Two suramin molecules were found to fit into the catalytic chamber (Fig. 2a-2b, and Fig. 3b).

\section{The interactions of suramin with SARS-CoV-2 RdRp}

One suramin molecule (suramin \#1) is fit into a cavity formed by conserved motif $\mathrm{G}$ and the $\mathrm{N}$ terminus of motif B (Fig. 4a and Fig. 3). The chemical structure of suramin has a two-fold symmetry with a urea linker at the center (Fig. 1a). The EM density map for this suramin is very clear defined but only for half of the suramin molecule without the urea linker (Fig. 4a and 4b). The key interactions of suramin \#1 with RdRp were summarized in Fig. 4c and Extended Data Table 2, including hydrogen bonds, charge interactions, and hydrophobic packing interactions with conserved RdRp residues, which restrain the naphthalene-trisulfonic acid head in a relative narrow cavity. Two out of the three sulfonates (positions 3 and 5) form hydrogen bonds with the side chains from N497, K500, R569 and Q573, and the main chain from N497, while the sulfonate at positions 1 points toward the solvent and forms only one hydrogen bond with the side chain of N496. The K577 side chain forms cation- $\pi$ stacking with the naphthalene ring, and also forms a hydrogen bond with the amide bond linker between the naphthalene and benzene rings. The amide bond linker between the benzene rings $C$ and $D$ forms a hydrogen bond with main chain NH of G590. In addition, the suramin \# 1 is in van der Waals contact with several residues, including L576, A580, A685, Y689 and 1758. The other suramin molecule (suramin \#2) is fit into the cavity near the catalytic active site formed by conserved motifs $A, C, E$, and F (Fig. $4 \mathrm{~b}$ and Fig. 3). Also, only half of suramin was observed in the structure with clear EM density map. The key interactions of suramin \#2 with RdRp were summarized in Fig. 4d and Extended Data Table 2, including hydrogen bonds, charge interactions, and hydrophobic packing interactions. Different from suramin \# 1, the sulfonate at positions 5 of suramin \# 2 points toward the solvent and forms only one hydrogen bond with the side chain of R555, while the other two sulfonates at positions 1 and 3 form hydrogen bonds with the side chains from K551, R553, R555 and R836, and the main chains from A550 and K551. Meanwhile the side chain of R555 also forms a hydrogen bond with the amide bond linker between the naphthalene and benzene rings. The R836 side chain forms cation- $\pi$ stacking with the benzene ring $\mathrm{C}$. The $\mathrm{NH}$ of the benzene ring $D$ forms a hydrogen bond with the side chain of D865. In addition, suramin \# 2 is 
in van der Waals contact with several residues, including H439, 1548, S549, A840, S861 and L862. Sequence alignment with RdRp from several viruses indicated that these suramincontacting residues are conserved (Extended Data Fig. 5), suggesting that suramin may be a general inhibitor of viral RdRp, which implies that suramin could be used as a drug for treating infections of these viruses.

\section{Inhibition mechanism of suramin to the SARS-CoV-2 RdRp}

Structural comparison of the RdRp-suramin complex with the remdesivir-bound RdRp complex reveal a clear mechanism of RdRp inhibition by suramin (Fig. 5a). If the base position of remdesivir was defined as +1 position, then the binding of the first suramin molecule occupies the same space of -1 to -3 positions of RNA template strand (suramin \#1 in Fig. 5b). The second suramin molecule at the active site occupies the space of the primer strand ranging from -4 to +1 positions (suramin \#2 in Fig. 5 c). The binding of these two suramin molecules would thus block the binding of the RNA template-primer duplex to the active site as well as the entry of nucleotide triphosphate into the catalytic site, which results in the direct inhibition of the RdRp catalytic activity. The direct inhibition mechanism of SARS-CoV-2 RdRp by suramin is different from the suramin-mediated inhibition of the norovirus RdRp, which also contained two binding sites for suramin binding ${ }^{16}$. In each site, only half of suramin molecule was found. Structural comparison of the SARS-CoV-2 RdRp with the norovirus RdRp reveals only one of the two suramin binding sites (suramin \#2) is partially overlapped (Extended Data Fig.6a, 6c and 6d). Both suramin binding sites in norovirus RdRp did not clash with the RNA strands but one of the suramin binding site is overlapping with the proposed nucleotide entry channel, thus indirectly blocking RdRp polymerization activity, a mechanism different from the direct blocking of the binding of the RNA template to the SARS-CoV-2 RdRp by suramin (Fig. 5). In addition, structural comparisons of the SARS-CoV-2 RdRp-suramin structure with the structures of the norovirus RdRp bound to other suramin derivatives show that the suramin and suramin derivatives bind to the RdRp with diverse conformations and orientations ${ }^{16,17}$ (Extended Data Fig.6b, 6e and 7), indicating the possibility for other suramin derivatives to inhibit the SARSCoV-2 RdRp as tested below.

\section{Inhibition SARS-CoV-2 RdRp by suramin derivatives}

Suramin derivatives have been explored for diverse applications including anti-cancers and antiparasites $^{10}$. To determine the structure-activity-relationship (SAR), we screened a set of 8 different suramin derivatives using in vitro RdRp primer extension assays (Fig. 6a and Extended 
Data Fig.1f). All 8 tested suramin derivatives showed efficient inhibition of RdRp activity (Extended Data Fig.1g). NF157, NF279, and NF449 are most potent inhibitors with $\mathrm{IC}_{50}$ of 0.05 $\mu \mathrm{M}$, which are about 5-fold more potent than the parent drug suramin (Fig. 6b). Cell-based assays showed that NF110 inhibited SARS-CoV-2 replication with $\mathrm{EC}_{50}$ of $2.87 \mu \mathrm{M}$ (Fig. $6 \mathrm{c}$ ), while NF157 and NF279 inhibited SARS-CoV-2 replication with $\mathrm{EC}_{50}$ of $\sim 10 \mu \mathrm{M}$. The $\mathrm{CC}_{50}$ values of all suramin derivatives are over $1000 \mu \mathrm{M}$, indicating their good safety window and their potential use for treating COVID-19. However, there is a 200 -fold separation between their biochemical potency in inhibiting RdRp activity and their potency in inhibiting viral replication in cell-based assays, suggesting the difficulties of these suramin derivatives to be uptake by host cells ${ }^{18}$. Future drug formulation such as with glycol chitosan-based nanoparticles ${ }^{19}$ may improve their bioavailability to lung tissues and their potency in inhibiting viral replication.

The COVID-19 pandemic has infected many more people and caused many more deaths than anyone could imagined since the outbreak of SARS-CoV-2 in December of 2019. The ongoing pandemic urgently press the need for effective vaccines and drug treatments. Suramin is a century-old drug that has been used for treating African sleepiness and showed activity against a number of viruses without a clear mechanism. In this paper, we showed that suramin is a direct potent inhibitor of the viral RdRp, the essential enzyme for the viral life cycle. The structure reveals that suramin binds to the active site of RdRp, blocking the binding of both strands of the template-primer RNA substrate and inhibiting the polymerase activity of the RdRp. Suramin derivatives also showed potent inhibition of the RdRp activity and blocked viral replication in cell-based assays. Together, these results uncovered the structural mechanism of the first non-nucleotide inhibitor of the SARS-CoV-2 RdRp and suggested the potential use of suramin for treating the SARS-CoV-2 infections. The structure and biochemical results presented in this paper also provided a rationale to develop suramin analogs as well as drug formulations to improve their potency and efficacy to inhibit SARS-CoV-2 replication. 


\section{References}

1. Gorbalenya, A.E. et al. Severe acute respiratory syndrome-related coronavirus: The species and its viruses - a statement of the Coronavirus Study Group. bioRxiv, 2020.02.07.937862 (2020).

2. Dong, E., Du, H. \& Gardner, L. An interactive web-based dashboard to track COVID-19 in real time. The Lancet Infectious Diseases (2020).

3. Sanche, S. et al. High Contagiousness and Rapid Spread of Severe Acute Respiratory Syndrome Coronavirus 2. Emerging Infectious Disease journa/ 26, 1470 (2020).

4. Brun, R., Blum, J., Chappuis, F. \& Burri, C. Human African trypanosomiasis. The Lancet 375, 148-159 (2010).

5. Hawking, F. Symposium on onchocerciasis III. Chemotherapy of onchocerciasis. Transactions of The Royal Society of Tropical Medicine and Hygiene 52, 109-111 (1958).

6. Ren, P. et al. The approved pediatric drug suramin identified as a clinical candidate for the treatment of EV71 infection-suramin inhibits EV71 infection in vitro and in vivo. Emerg Microbes Infect 3, e62 (2014).

7. Albulescu, I.C., Kovacikova, K., Tas, A., Snijder, E.J. \& van Hemert, M.J. Suramin inhibits Zika virus replication by interfering with virus attachment and release of infectious particles. Antiviral Res 143, 230-236 (2017).

8. Albulescu, I.C. et al. Suramin inhibits chikungunya virus replication through multiple mechanisms. Antiviral Research 121, 39-46 (2015).

9. Henß, L. et al. Suramin is a potent inhibitor of Chikungunya and Ebola virus cell entry. Virol J 13, 149 (2016).

10. Wiedemar, N., Hauser, D.A. \& Maser, P. 100 Years of Suramin. Antimicrob Agents Chemother 64(2020).

11. Salgado-Benvindo, C. et al. Suramin Inhibits SARS-CoV-2 Infection in Cell Culture by Interfering with Early Steps of the Replication Cycle. Antimicrob Agents Chemother 64(2020).

12. Subissi, L. et al. One severe acute respiratory syndrome coronavirus protein complex integrates processive RNA polymerase and exonuclease activities. Proc Natl Acad Sci U S A 111, E3900-9 (2014).

13. Kirchdoerfer, R.N. \& Ward, A.B. Structure of the SARS-CoV nsp12 polymerase bound to nsp7 and nsp8 co-factors. Nat Commun 10, 2342 (2019).

14. Yin, W. et al. Structural basis for inhibition of the RNA-dependent RNA polymerase from SARS-CoV2 by remdesivir. Science 368, 1499-1504 (2020).

15. Wang, M. et al. Remdesivir and chloroquine effectively inhibit the recently emerged novel coronavirus (2019-nCoV) in vitro. Cell Res 30, 269-271 (2020).

16. Mastrangelo, E. et al. Structure-Based Inhibition of Norovirus RNA-Dependent RNA Polymerases. Journal of Molecular Biology 419, 198-210 (2012).

17. Croci, R. et al. Structural bases of norovirus RNA dependent RNA polymerase inhibition by novel suramin-related compounds. PloS one 9, e91765-e91765 (2014).

18. Alsford, S., Field, M.C. \& Horn, D. Receptor-mediated endocytosis for drug delivery in African trypanosomes: fulfilling Paul Ehrlich's vision of chemotherapy. Trends Parasito/29, 207-12 (2013).

19. Cheng, B., Gao, F., Maissy, E. \& Xu, P. Repurposing suramin for the treatment of breast cancer lung metastasis with glycol chitosan-based nanoparticles. Acta Biomater 84, 378-390 (2019).

20. Zheng, S.Q. et al. MotionCor2: anisotropic correction of beam-induced motion for improved cryoelectron microscopy. Nat Methods 14, 331-332 (2017).

21. Rohou, A. \& Grigorieff, N. CTFFIND4: Fast and accurate defocus estimation from electron micrographs. J Struct Bio/ 192, 216-21 (2015).

22. Zivanov, J. et al. New tools for automated high-resolution cryo-EM structure determination in 
RELION-3. Elife 7(2018).

23. Punjani, A., Rubinstein, J.L., Fleet, D.J. \& Brubaker, M.A. cryoSPARC: algorithms for rapid unsupervised cryo-EM structure determination. Nat Methods 14, 290-296 (2017).

24. Rosenthal, P.B. \& Henderson, R. Optimal determination of particle orientation, absolute hand, and contrast loss in single-particle electron cryomicroscopy. J Mol Bio/ 333, 721-45 (2003).

25. Pettersen, E.F. et al. UCSF chimera - A visualization system for exploratory research and analysis. Journal of Computational Chemistry 25, 1605-1612 (2004).

26. Emsley, P. \& Cowtan, K. Coot: model-building tools for molecular graphics. Acta Crystallographica Section D-Biological Crystallography 60, 2126-2132 (2004).

27. Adams, P.D. et al. PHENIX: a comprehensive Python-based system for macromolecular structure solution. Acta Crystallogr D Biol Crystallogr 66, 213-21 (2010).

28. Chen, V.B. et al. MolProbity: all-atom structure validation for macromolecular crystallography. Acta Crystallogr D Biol Crystallogr 66, 12-21 (2010).

29. Goddard, T.D. et al. UCSF ChimeraX: Meeting modern challenges in visualization and analysis. Protein Sci 27, 14-25 (2018).

30. Saez-Alvarez, Y., Arias, A., Del Aguila, C. \& Agudo, R. Development of a fluorescence-based method for the rapid determination of Zika virus polymerase activity and the screening of antiviral drugs. Sci Rep 9, 5397 (2019).

\section{Acknowledgments}

The cryo-EM data were collected at the Cryo-Electron Microscopy Research Center, Shanghai Institute of Materia Medica. This work was partially supported by National Key R\&D Program of China (2020YFC0861000), CAMS Innovation Fund for Medical Sciences No. 2020-I2M-CoV19001, and Tsinghua University-Peking University Center for Life Sciences 045-160321001 to S. Z.; the National Key R\&D Programs of China 2018YFA0507002; Shanghai Municipal Science and Technology Major Project 2019SHZDZX02 and XDB37030103 to H.E.X.; the 100 Talents Program of the Chinese Academy of Sciences, Chinese Academy of Sciences grant (XDA12010317), Natural Science Foundation of Shanghai (18ZR1447700) to X.Y; the National Natural Science Foundation of China (31900869) and Shanghai Sailing Program (19YF1456800) to Z.L; Science and Technology Commission of Shanghai Municipal 20431900100 and Jack Ma Foundation 2020-CMKYGG-05 to H.J.; National Natural Science Foundation 31770796, National Science and Technology Major Project2018ZX09711002, and K.C. Wong Education Foundation to Y.J, the National Natural Science Foundation of China (31970165) to L. Z.; the National Natural Science Foundation of China (81903433) to J. S..

Author contributions: W.Y. designed the expression constructs, purified the RdRp complex, prepared samples and cryo-EM grids, performed data collection and processing toward the structure determination, analyzed the structures, and prepared Fig.S and manuscript. X.L. designed RdRp activity assays and discovered RdRp inhibition by suramin, participated in data 
interpretation and Fig. preparation; Z.L., Z.Z., Q. W., and X.Y. evaluated the specimen by negative-stain EM, screened the cryo-EM conditions, prepared the cryo-EM grids and collected cryo-EM images, performed data processing, density map calculations, model building, and Fig. preparation; L.Z., Q.W., and G.X. designed and performed cell-based viral inhibition assays; X. W., F. Z., and M.G. participated in expression, purification and functional assays of the RdRp; J. S., E. Y. and M. L. participated in the analysis of suramin derivatives; Y.J. participated in experimental design and manuscript editing; H.J. conceived and coordinated the project; S.Z. conceived the project, initiated collaboration with H.E.X., and supervised X.L.; H.E.X. conceived and supervised the project, analyzed the structures, and wrote the manuscript with inputs from all authors.

Competing interests: The authors declare no competing interests.

Data and materials availability: Density maps and structure coordinates have been deposited with immediate release. The accession numbers of Electron Microscopy Database and the Protein Data Bank are EMD-30572and PDB ID 7D4F for the SARS-CoV-2 RdRp bound to suramin. These files are immediately available for download as supplemental materials in this article. Materials are available up on request. 


\section{Figure Legends:}

Fig. 1. Inhibition of RdRp by suramin and the potential anti-SARS-CoV-2 effect of suramin

a. The chemical structure of suramin.

b. The 30-base template and 20-base primer duplex RNAs with a FAM at the 5' of the primer. Poly-A was used in the gel-based elongation assay, while Poly- $U$ was used in the fluorescencebased activity assay for SARS-CoV-2 RdRp.

c. Elongation of partial RNA duplex by the purified RdRp complex and its inhibition by suramin. The EP means the elongated product, while the P means the primer RNA strand.

d. Gel mobility shift of the RdRp-RNA complex and the effect of suramin.

e. $\mathrm{IC}_{50}$ of suramin for RdRp complex.

f. $\mathrm{EC}_{50}$ of suramin for SARS-CoV-2 inhibition and $\mathrm{CC}_{50}$ of suramin for cell-based toxicity.

\section{Fig. 2. Overall Structures of the RdRp-suramin complex}

a. Two views of cryo-EM density map of the RdRp-suramin complex, SARS-CoV2 nsp12 contains a N-terminal extension composed of the $\beta$-hairpin (green), the NiRAN domain (yellow) and an interface domain (purple) adjacent to the RdRp core domain, which contains subdomains: fingers, palm, and thumb, colored with violet, orange, and blue, respectively. The nsp12 binds to a heterodimer of nsp7 (light green) and nsp8 (nsp8-2, red) as well as to a second subunit of nsp8 (nsp-1, red). The two bound suramin molecules are set as dark green. Color code is used throughout.

b. Two views of the overall structure of the RdRp-suramin complex, the color scheme is according as above.

Fig. 3. The conserved motif A-G in the RdRp-suramin complex.

a. Schematic diagram for the components of the RdRp complex subunit nsp12, the motif (A to $G$ ) are highlighted.

b. The color code is as follows: motif A (cyan), motif B (gold), motif C (orange red), motif D (medium violet red), motif $E$ (magenta), motif $F$ (blue), and motif $G$ (green).

\section{Fig. 4. Close views of the interactions between the SARS-CoV-2 RdRp and suramin} a-b. The maps for the two suramin molecules.

c-d. Interactions of the two suramin molecules with RdRp. The hydrogen bond is displayed as green dashed line. 
Fig. 5. Inhibition mechanism from comparison with the remdesivir-bound RdRp structure a. The two overall views of the RdRp-suramin complex overlapped with the remdesivir-bound RdRp structure (PDB ID: 7BV2). For clarity, only the polymerase domains are presented. The remdesivir bound RdRp structure is set as light gray, the template RNA is set as cyan, and the primer RNA is set as red.

b. Close view of suramin \#1 overlapped with RNA template strand.

c. Close view of suramin \#2 overlapped with RNA primer strand.

Fig. 6. Inhibition SARS-CoV-2 RdRp by suramin derivatives.

a. The chemical structures of suramin derivatives.

b. The inhibition of RdRp activity by suramin derivatives.

c. Inhibition of viral replication and cellular toxicity of suramin derivatives. 


\section{Supplementary Material}

\section{Materials and Methods}

\section{Constructs and expression of the RdRp complex}

The RdRp complex was prepared according to same method reported ${ }^{13}$ as described below. The full-length gene of the SARS-CoV-2 nsp12(residues 1-932) was chemically synthesized with codon optimization (General Biosystems). The gene was cloned into a modified pFastBac baculovirus expression vector containing a 5' ATG starting sequence and C-terminal Tobacco Etch Virus (TEV) protease site followed by a His8 tag. The plasmid contains an additional methionine at the N-terminus and GGSENLYFQGHHHHHHHH at the C-terminus of nsp12. The full-length genes for nsp7 (residues 1-83) and nsp8 (residues 1-198) were cloned into the pFastBac vector containing a 5' ATG starting sequence. All constructs were generated using the Phanta Max Super-Fidelity DNA Polymerase (Vazyme Biotech Co.,Ltd) and verified by DNA sequencing. All constructs were expressed in Spodoptera frugiperda (Sf9) cells. Cell cultures were grown in ESF 921 serum-free medium (Expression Systems) to a density of 2-3 million cells per $\mathrm{ml}$ and then infected with three separate baculoviruses at a ratio of 1:2:2 for nsp12, nsp7 and nsp8 at a multiplicity of infection (m.o.i.) of about 5. The cells were collected $48 \mathrm{~h}$ after infection at $27^{\circ} \mathrm{C}$ and cell pellets were stored at $-80^{\circ} \mathrm{C}$ until use.

In addition, the genes of nsp7 and nsp8 were cloned into a modified pET-32a (+) vector containing a 5' ATG starting sequence and C-terminal His8 tag with a TEV cleavage site for expression in E. coli. Plasmids were transformed into BL21(DE3) (Invitrogen). Bacterial cultures were grown to an OD600 of 0.6 at $37^{\circ} \mathrm{C}$, and then the expression was induced with a final concentration of $0.1 \mathrm{mM}$ of isopropyl $\beta-D$-1-thiogalactopyranoside (IPTG) and the growth temperature was reduced to $16{ }^{\circ} \mathrm{C}$ for $18-20 \mathrm{~h}$. The bacterial cultures were pelleted and stored at $-80^{\circ} \mathrm{C}$ until use.

\section{Purification of the RdRp complex}

The purification of nsp7 and nsp8 expressed in bacteria was similar to the purification of nsp7 and nsp8 reported previously ${ }^{13}$. Briefly, bacterial cells were lysed with a high-pressure homogenizer operating at 800 bar. Lysates were cleared by centrifugation at $25,000 \times \mathrm{g}$ for $30 \mathrm{~min}$ and were then bound to Ni-NTA beads (GE Healthcare). After wash with buffer containing $50 \mathrm{mM}$ imidazole, the protein was eluted with buffer containing $300 \mathrm{mM}$ imidazole. The tag was removed with incubation of TEV protease overnight and protein samples were concentrated with $3 \mathrm{kDa}$ or $30 \mathrm{kDa}$ molecular weight cut-off centrifuge filter units (Millipore 
Corporation) and then size-separated by a Superdex 75 Increase10/300 GL column in $25 \mathrm{mM}$ HEPES pH 7.4, 200 mM sodium chloride, 5\% (v/v) glycerol. The fractions for the nsp7 or nsp8 were collected, concentrated to about $10 \mathrm{mg} / \mathrm{ml}$, and stored at $-80^{\circ} \mathrm{C}$ until use.

The insect cells containing the co-expressed RdRp complex were resuspended in binding buffer of $25 \mathrm{mM}$ HEPES pH 7.4, $300 \mathrm{mM}$ sodium chloride, $25 \mathrm{mM}$ imidazole, $1 \mathrm{mM}$ magnesium chloride, 0.1\% (v/v) IGEPALCA-630 (Anatrace), $1 \mathrm{mM}$ tris (2-carboxyethyl) phosphine (TCEP), $10 \%(v / v)$ glycerol with additional EDTA-free Protease Inhibitor Cocktail (Bimake), and then incubated with agitation for $20 \mathrm{~min}$ at $4{ }^{\circ} \mathrm{C}$. The incubated cells were lysed with a high-pressure homogenizer operating at 500 bar. The supernatant was isolated by centrifugation at $30,000 \times g$ for $30 \mathrm{~min}$, followed by incubation with Ni-NTA beads (GE Healthcare) for $2 \mathrm{~h}$ at $4^{\circ} \mathrm{C}$. After binding, the beads were washed with 20 column volumes of wash buffer of $25 \mathrm{mM} \mathrm{HEPES}, \mathrm{pH}$ 7.4, $300 \mathrm{mM}$ sodium chloride, $25 \mathrm{mM}$ imidazole, $1 \mathrm{mM}$ magnesium chloride, $1 \mathrm{mM}$ TCEP, and $10 \%$ (v/v) glycerol. The protein was eluted with 3-4 column volumes of elution buffer of $25 \mathrm{mM}$ HEPES pH 7.4, 300 mM sodium chloride, 300 mM imidazole, $1 \mathrm{mM}$ magnesium chloride, $1 \mathrm{mM}$ TCEP, and $10 \%(\mathrm{v} / \mathrm{v})$ glycerol.

The co-expressed RdRp complex was incubated with additional nsp7 and nsp8 from the bacterial expression in a 1:1:2 molar ratios and incubated at $4{ }^{\circ} \mathrm{C}$ for $4 \mathrm{~h}$. Incubated $\mathrm{RdRp}$ complex was concentrated with a $100 \mathrm{kDa}$ molecular weight cut-off centrifugal filter unit (Millipore Corporation) and then size-separated by a Superdex 200 Increase 10/300 GL column in $25 \mathrm{mM}$ HEPES pH 7.4, $300 \mathrm{mM}$ sodium chloride, $1 \mathrm{mM}$ magnesium chloride, $1 \mathrm{mM}$ TCEP. The fractions for the monomeric complex were collected and concentrated to up to $12 \mathrm{mg} / \mathrm{ml}$. Suramin sodium salt (purchased from MedChemExpress) was dissolved in water up to $50 \mathrm{mM}$. Suramin derivatives (purchased from TopScience Co., Ltd) was dissolved in water at concentrations of 5 to $50 \mathrm{mM}$. For the suramin bound complex, the concentrated RdRp complex at the concentration of $12 \mathrm{mg} / \mathrm{ml}$ were incubated with $0.8 \mathrm{mM}$ suramin at $4{ }^{\circ} \mathrm{C}$ for $0.5 \mathrm{~h}$ for the next step of EM experiments.

\section{Cryo-EM sample preparation and data acquisition}

An aliquot of $3 \mu \mathrm{L}$ protein sample of suramin-bound complex $(12 \mathrm{mg} / \mathrm{mL})$ containing $0.0035 \%$ DDM was applied onto a glow-discharged 200 mesh grid (Quantifoil R1.2/1.3), blotted with filter paper for $3.0 \mathrm{~s}$ and plunge-frozen in liquid ethane using a FEI Vitrobot Mark IV. Cryo-EM micrographs were collected on a 300kV Titan Krios microscope (FEI) equipped with a Gatan 
image filter (operated with a slit width of $20 \mathrm{eV}$ ) (GIF) and K3 direct detection camera. The microscope was operated at a calibrated magnification of $46,773 \mathrm{X}$, yielding a pixel size of $1.069 \AA$ on micrographs. 11,846 micrographs in total were collected at an electron dose rate of $22.7 \mathrm{e}^{-} / \AA^{2} \cdot \mathrm{s}$ with a defocus range of $-0.5 \mu \mathrm{m}$ to $-2.0 \mu \mathrm{m}$. Each movie with an accumulated dose of $68 \mathrm{e}^{-/ \AA^{2}}$ on sample were fractionated into a movie stack of 36 image frames.

\section{Image processing}

Frames in each movie stack were aligned for beam-induced motion correction using the program MotionCorr220. CTFFIND421 was used to determine the contrast transfer function (CTF) parameters. 10,241 good micrographs were selected for further data processing. Auto-picking program of Relion $3.0^{22}$ was used to pick the particles with the model of the apo RdRp complex of COVID-19 (PDB ID: 7BV1) ${ }^{14}$ as a reference, yielding a total of 8,557,180 picked particles. Then, the extracted particle stack was transferred to software Cryosparc v2 ${ }^{23}$ and a round of reference-free 2D classification was performed. 3,159,808 particles were selected from classes representing projections of suramin-bound RdRp complex in different orientations, and subjected to two rounds of Heterogenous Refinement using a reconstruction of the apo RdRp complex of COVID-19 (EMD-30209) ${ }^{14}$ as a starting map. One converged 3D class with highresolution feature contains one nsp12, one nsp7 and two copies of nsp8. The particles from that 3D class were then imported back into Relion 3.0 and subjected to a round of focused alignment with a mask including the whole protein components. Finally, 95,845 particles from a 3D class showing the highest resolution feature were selected for a round of 3D refinement. After a round of CTF refinement and Bayesian polishing of particles, another round 3D refinement was performed, yielding a final reconstruction at a global resolution of $2.57 \AA$ based on the goldstandard Fourier shell correlation $(F S C)=0.143$ criterion $^{24}$. The local resolution was calculated with Relion 3.0.

\section{Model building}

The model of suramin-bound RdRp complex was built by docking the model of apo structure of COVID-19 RdRp (PDB ID:7BV1) into the density map using UCSF Chimera ${ }^{25}$, followed by ab initio model building of the N-terminal NiRAN domain of nsp12 and one copy of nsp8 in COOT ${ }^{26}$, and real space refinement using real_space_refine program in PHENIX ${ }^{27}$. The model statistics were calculated with MolProbity ${ }^{28}$ and listed in Extended Data Table 1. Structural Fig.s were prepared in Chimera or ChimeraX ${ }^{29}$. 


\section{Preparation of template-primer RNA for polymerase assays}

For the poly-A template-primer RNA, a short RNA oligonucleotide with sequence of 5'-FAMGCUAUGUGAGAUUAAGUUAU-3' (Sangon Biotech Co., Ltd) was used as the primer strand and a longer RNA oligonucleotide with sequence of 5'-

AAAAAAAAAAAUAACUUAAUCUCACAUAGC -3' (Sangon Biotech Co., Ltd) was used as template strand. To anneal the RNA duplex, both oligonucleotides were mixed at equal molar ratio in annealing buffer (10 $\mathrm{mM}$ Tris- $\mathrm{HCl}, \mathrm{pH} 8.0,25 \mathrm{mM} \mathrm{NaCl}$ and $2.5 \mathrm{mM}$ EDTA), denatured by heating to $94{ }^{\circ} \mathrm{C}$ for $5 \mathrm{~min}$ and then slowly cooled to room temperature. The poly-U templateprimer RNA was prepared similar to poly-A with the sequences of 5'-FAM-

GCUAUGUGAGAUUAAGUUAU-3' and 5'- UUUUUUUUUUAUAACUUAAUCUCACAUAGC -3'.

\section{Gel mobility shift assay to detect RNA-RdRp protein binding}

A gel mobility shift assay was performed to detect the effect of tested compounds on RNA binding by the RdRp complex. The binding reaction contained $20 \mathrm{mM}$ Tris- $\mathrm{HCl} 8.0,10 \mathrm{mM} \mathrm{KCl}$, $6 \mathrm{mM} \mathrm{MgCl}_{2}, 0.01 \%$ Triton-X100, 1mM DTT, $1.14 \mathrm{U} / \mathrm{ul}$ RNase Inhibitor(Vazyme Biotech Co.,Ltd), $9 \mu \mathrm{g}$ RdRp complex protein with1 $\mu \mathrm{g}$ poly-A template-primer RNA, and increasing amounts of corresponding compounds $(0,1,10,100,1000$ and $5000 \mu \mathrm{M}$ for suramin, while $0,1,10,100$, 1000, 5000 and $10000 \mu \mathrm{M}$ for RDV-TP). Binding reactions were incubated for $30 \mathrm{~min}$ at room temperature and resolved on 4-20\% native polyacrylamide gel (Thermo Fisher) running in $1 \times$ TBE buffer at $90 \mathrm{~V}$ for $1.5 \mathrm{~h}$ in $4{ }^{\circ} \mathrm{C}$ cool-room. The gel was imaged with a Tanon-5200 Multi Fluorescence Imager according to the manufacturer's protocol.

\section{RdRp enzymatic activity assay and its inhibition by suramin}

The purified SARS-CoV-2 RdRp complex from insect cell at final concentration of $1 \mu \mathrm{M}$ was incubated with $3.0 \mu \mathrm{M}$ poly-A template-primer RNA and $10 \mathrm{mM}$ UTP(Macklin) in the presence of $1.14 \mathrm{U} / \mu \mathrm{l}$ RNase inhibitor in reaction buffer containing $20 \mathrm{mM}$ Tris, pH8.0, $10 \mathrm{mM} \mathrm{KCl,6mM}$ $\mathrm{MgCl}_{2}, 0.01 \%$ Triton-X100, and $1 \mathrm{mM}$ DTT, which were prepared with DEPC-treated water. The total reaction volume was $20 \mu \mathrm{l}$. After incubation for $60 \mathrm{~min}$ at a $37^{\circ} \mathrm{C}$ water bath, $40 \mu \mathrm{l}$ quench buffer (94\% formamide, 30 mM EDTA, prepare with DEPC-treated water) was added to stop the reaction. A sample of $18 \mu \mathrm{l}$ of reaction was mixed with $2 \mu \mathrm{l}$ of $10 x$ DNA loading buffer (Solarbio). Half of the sample $(10 \mu \mathrm{l})$ was loaded onto a $10 \%$ Urea-PAGE denatured gel, run at $120 \mathrm{~V}$ for 1h, and imaged with a Tanon-5200 Multi Fluorescence Imager. The setup for the inhibition assays of the RdRp by suramin is identical to the above for the RdRp enzymatic assays, except 
that suramin was added to final concentrations of $0,1,2,4,8,16,32 \mu \mathrm{M}$ for 60 min before the addition of $10 \mathrm{mM}$ UTP.

\section{Fluorescence-based activity assay for SARS-CoV-2 RdRp}

The detection of RNA synthesis by SARS-CoV-2 RdRp were established based on a real-time assay with the fluorescent dye SYTO 9 (Thermo Fisher), which binds dsRNA but not sSRNA template molecules. The fluorescence emitted was recorded in real-time using a TECAN F200 with excitation and emission filters at 485 and $520 \mathrm{~nm}$, respectively. The assay records the synthesis of dsRNA in a reaction using a poly- $U$ molecule as a template and ATP as the nucleotide substrate, which has been adapted from methods previously documented for the detection of Zika virus polymerase activity ${ }^{30}$. Reactions were performed in individual wells of black 384-well low volume round bottom plates. The standard reaction contained $20 \mathrm{mM}$ Tris$\mathrm{HCl}, \mathrm{pH}$ 8.0, $10 \mathrm{mM} \mathrm{KCl}, 6 \mathrm{mM} \mathrm{MgCl}_{2}, 180 \mu \mathrm{M}$ ATP, $0.2 \mu \mathrm{M}$ poly-U template-primer RNA, 0.01\%Trition-X100,1 mM DTT,0.025 U/ml RNase inhibitor (Vazyme Biotech Co., Ltd), and 0.25 $\mu \mathrm{M}$ SYTO 9 (50 $\mu \mathrm{M}$ stock solution in TE buffer $\mathrm{pH}$ 7.5). The assay was initiated by the addition of $5 \mu \mathrm{g} / \mathrm{ml}$ SARS-CoV-2 RdRp and the fluorescence was recorded over $30 \mathrm{~min}$ at room temperature. The reaction with equivalent of DMSO was set as MAX control, while the reaction with no SARS-CoV-2 RdRp was set as MIN control. The reactions were carried out in the presence of $0.2 \mu \mathrm{M}$ poly-U template-primer RNA and $180 \mu \mathrm{M}$ ATP, and increasing concentrations of each inhibitor. Fluorometric results were expressed as mean $\pm \mathrm{SD}$. Statistical significance was analyzed by two-way ANOVA using GraphPad Prism, version 8, as specified in the Fig. legends. $\mathrm{Km}$ determinations were obtained by plotting the velocity of the reaction as a function of nucleotide or ssRNA template concentrations using nonlinear regression. $I_{50}$ values were obtained by fitting the velocity data to a four-parameter logistic equation. Kinetic parameters and $\mathrm{IC}_{50}$ values were calculated using Sigmaplot, version 11.

\section{Vero E6 cell based antiviral assay for suramin and suramin derivatives}

African green monkey kidney Vero E6 cell line was obtained from American Type Culture Collection (ATCC, no. 1586) and maintained in Dulbecco's Modified Eagle Medium (DMEM; Gibco Invitrogen) supplemented with 10\% fetal bovine serum (FBS; Gibco Invitrogen), 1\% antibiotic/antimycotic (Gibco Invitrogen), at $37^{\circ} \mathrm{C}$ in a humidified $5 \% \mathrm{CO}_{2}$ incubator. A clinical isolate of SARS-CoV-2 (nCoV-2019BetaCoV/Wuhan/WIV04/2019) was propagated in Vero E6 cells, and viral titer was determined as described previously ${ }^{15}$. All the infection experiments were performed at biosafety level-3 (BSL-3). 
Pre-seeded Vero E6 cells ( $5 \times 10^{4}$ cells/well) were incubated with the different concentration of the indicated compounds for 1 hour, and then were infected with SARS-CoV-2 at a MOI of 0.01. Two hours later, the virus-drug mixture was removed and cells were further cultured with a fresh compound containing medium. At $24 \mathrm{~h}$ p.i.,., we measured viral RNA copy number in cell supernatant using real time PCR, as described previously ${ }^{15}$. DMSO was used in the controls. At least two independent experiments were carried out for each compound.

\section{The cytotoxicity assay}

The cytotoxicity of the tested drugs on Vero E6 were determined by CCK8 assays (Beyotime, China).

\section{Statistical analysis}

The $I_{50}$ and $\mathrm{EC}_{50}$ values were expressed as mean $\pm \mathrm{SD}$ from three independent experiments and determined via the nonlinear regression analysis using GraphPad Prism software 8.0 (GraphPad Software, Inc., San Diego, CA, USA)

\section{Extended Data Fig.s}

\section{Extended Data Fig.1. Purification and characterization of the RdRp complex}

a. Gel filtration profile of the RdRp complex with additional nsp7 and nsp8, showing a sharp peak.

b. SDS gel of the purified RdRp complex with additional nsp7 and nsp8, showing balanced ratios for each subunit.

c. Gel mobility shift of the RdRp-RNA complex and the effect of RDV-TP.

d. IC 50 of RDV-TP for the RdRp complex.

e. EC50 of remdesivir for SARS-CoV-2 inhibition and CC50 of remdesivir for cell-based toxicity.

f. The structures of PPADS and iso-PPADS.

g. Elongation of partial RNA duplex by the purified RdRp complex and its inhibition by 9 suramin derivatives at $0.5-5.0 \mathrm{mM}$ concentrations depending on the solubility of each compound.

\section{Extended Data Fig.2. Single particle cryo-EM analysis of the RdRp-suramin complex.}

a. Representative cryo-EM micrograph of the RdRp-suramin complex.

b. Representative 2D class averages of the RdRp-suramin complex. 
c. Fourier shell correlation curves of cryo-EM map for the the suramin-RdRp complex.

d. Euler angle distribution of particles used in the final reconstruction.

e. Flowchart of cryo-EM works of the suramin-RdRp complex with maps colored by local resolution $(\AA)$.

\section{Extended Data Fig.3. Cryo-EM map for the RdRp in complex with suramin.}

a-g. Cryo-EM map and model of nsp12 (residues 111-116) (A), nsp8-1 (residues 127-132) (B), nsp12 (residues 169-199) (C), nsp7 (residues 26-40) (D), nsp8-2 (residues 85-95) (E), and the two bound suramin molecules ( $F$ and $G)$.

\section{Extended Data Fig.4. The structural comparison of the RdRp-suramin complex with the apo RdRp complex.}

The two overall views of the RdRp-suramin complex overlapped with the apo RdRp structure (PDB ID: 7BV1). For clarity, only the polymerase domains are retained. The apo RdRp structure is set as light gray.

\section{Extended Data Fig.5. Sequence alignment of 12 coronavirus nsp12.}

Sequence alignment of nsp12 from eight $\beta$-CoVs (SARS-CoV-2, SARS-CoV, MERS-CoV, RaTG13, PCoV, MHV, HKU1, HCoV-OC43), one Y-CoVs (IBV) and three a-CoVs (HCoV-NL63, HCoV-229E, TGEV). Black arrows mark the residues interacting with suramin \#1, blue arrows mark the residues interacting with suramin \#2, while the red box sign the residues interacting with suramin molecules by hydrogen bonds.

\section{Extended Data Fig.6. Comparison of SARS-CoV-2 RdRp-suramin complex with MNV (Murine Noroviruses) RdRp-suramin complex (PDB ID: 3UR0) and MNV RdRp-NF023(PDB ID: 3URF).}

a. Superimposition of the SARS-CoV-2 RdRp-suramin structure with the MNV RdRp-suramin structure based on the polymerase domain. Only the polymerase domain of SARS-CoV-2 RdRp is retained.

b. Superimposition of SARS-CoV-2 RdRp-suramin structure with MNV RdRp-NF023 structure based on the polymerase domain.

c. A close view of the suramin within the catalytic site in the MNV RdRp-suramin structure.

d. A close view of the suramin within the catalytic site in the SARS-CoV-2 RdRp-suramin structure. 
e. A close view of the NF023 within the catalytic site in the MNV RdRp- NF023 structure.

Extended Data Fig.7. Comparison of SARS-CoV-2 RdRp-suramin complex with MNV RdRp-suramin derivative 6 complex (PDB ID: 4NUR) and HNV (Human Noroviruses) RdRp-suramin derivative 6 (PDB ID: 4NRT).

a. Superimposition of SARS-CoV-2 RdRp-suramin structure with MNV RdRp-suramin derivative 6 structure based on the polymerase domain. Only the polymerase domain of SARS-CoV-2 $\mathrm{RdRp}$ is retained.

b. Superimposition of SARS-CoV-2 RdRp-suramin structure with HNV RdRp-suramin derivative 6 structure based on the polymerase domain.

c. A close view of the suramin derivative 6 within the active site in the MNV RdRp-suramin derivative 6 structure.

d. A close view of the suramin within the active site in the SARS-CoV-2 RdRp-suramin structure.

e. A close view of the suramin derivative 6 within the active site in the HNV RdRp-suramin derivative 6 structure.

Extended Data Table 1, EM Data and Structure Refinement Statistics.

Extended Data Table 2, Interactions between suramin and nsp12 in the RdRp-suramin structure. 
a<smiles>[3H]C=C(NC(=O)c1ccc(C)c(NC(=O)c2cccc(NC(=O)Nc3cccc(C(=O)Nc4cc(C(=O)Nc5[Z7]ccc(S(=O)(=O)[O-])c5)ccc4C)c3)c2)c1)c1c(S(=O)(=O)[O-])cc(S(=O)(=O)[O-])cc1S(=O)(=O)[O-]</smiles>

Suramin

b

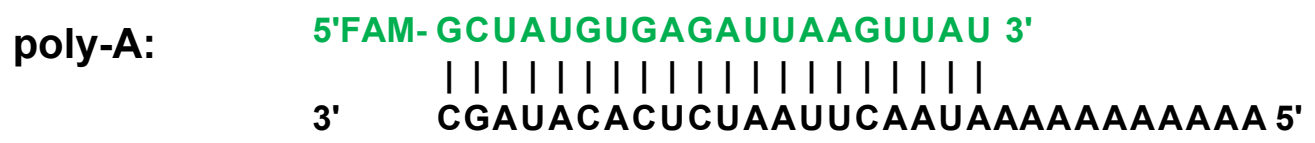
poly-U: 5'FAM- GCUAUGUGAGAUUAAGUUAU 3'

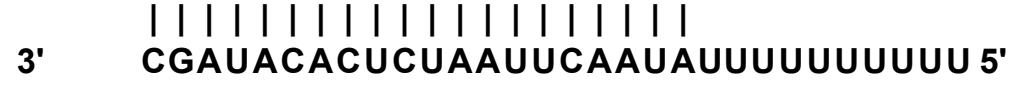

C
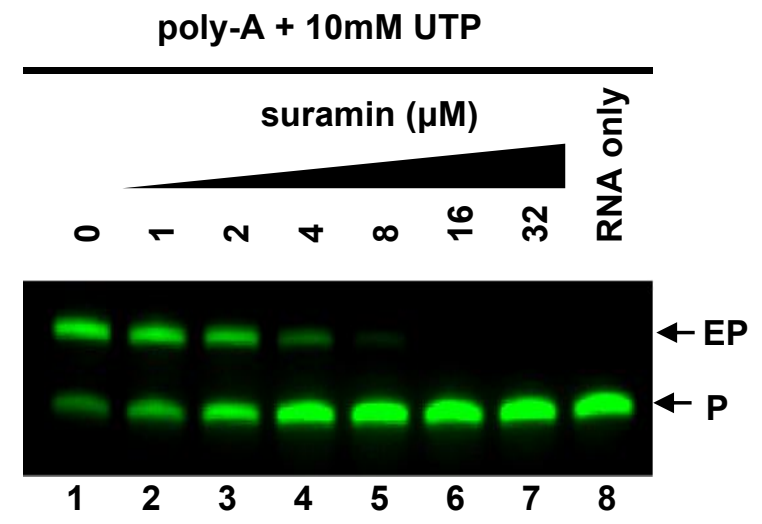

e

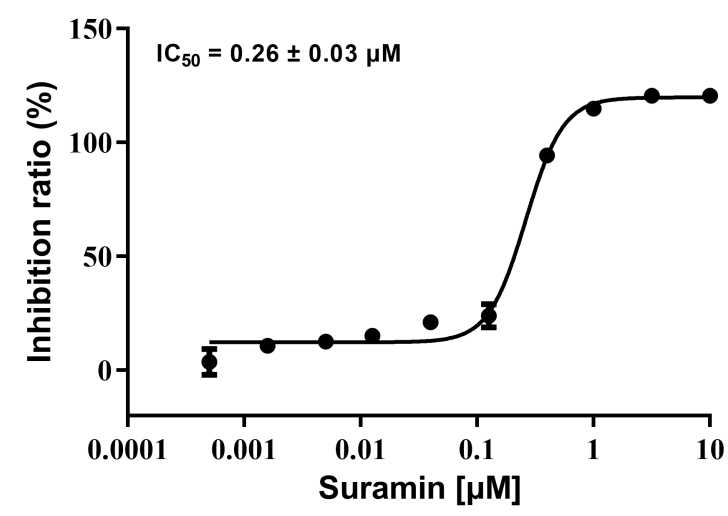

d

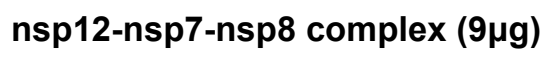
+ poly-A $(1 \mu \mathrm{g})$

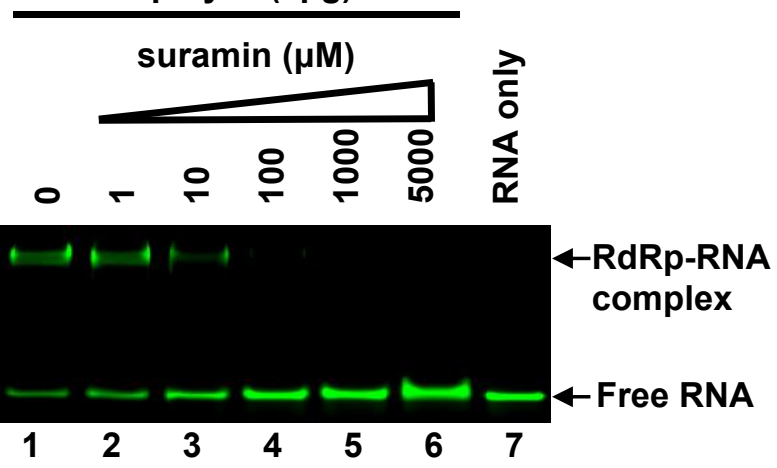

f

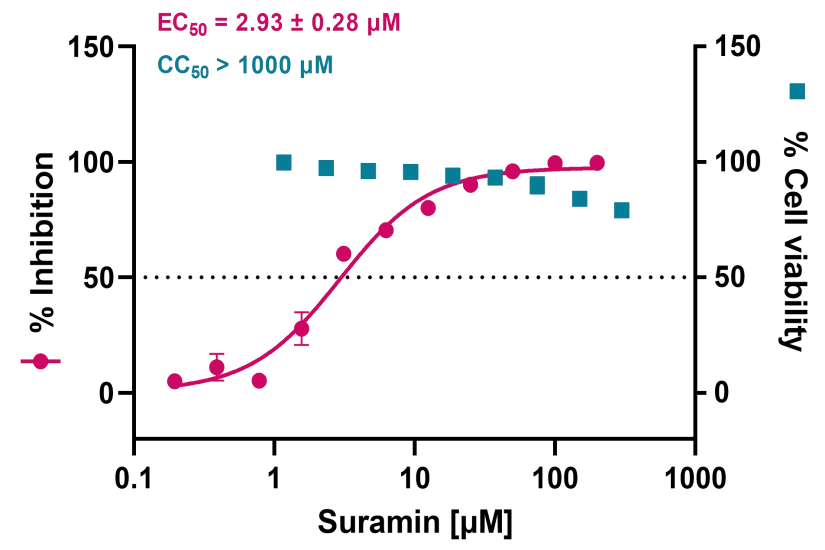


Figure 2.

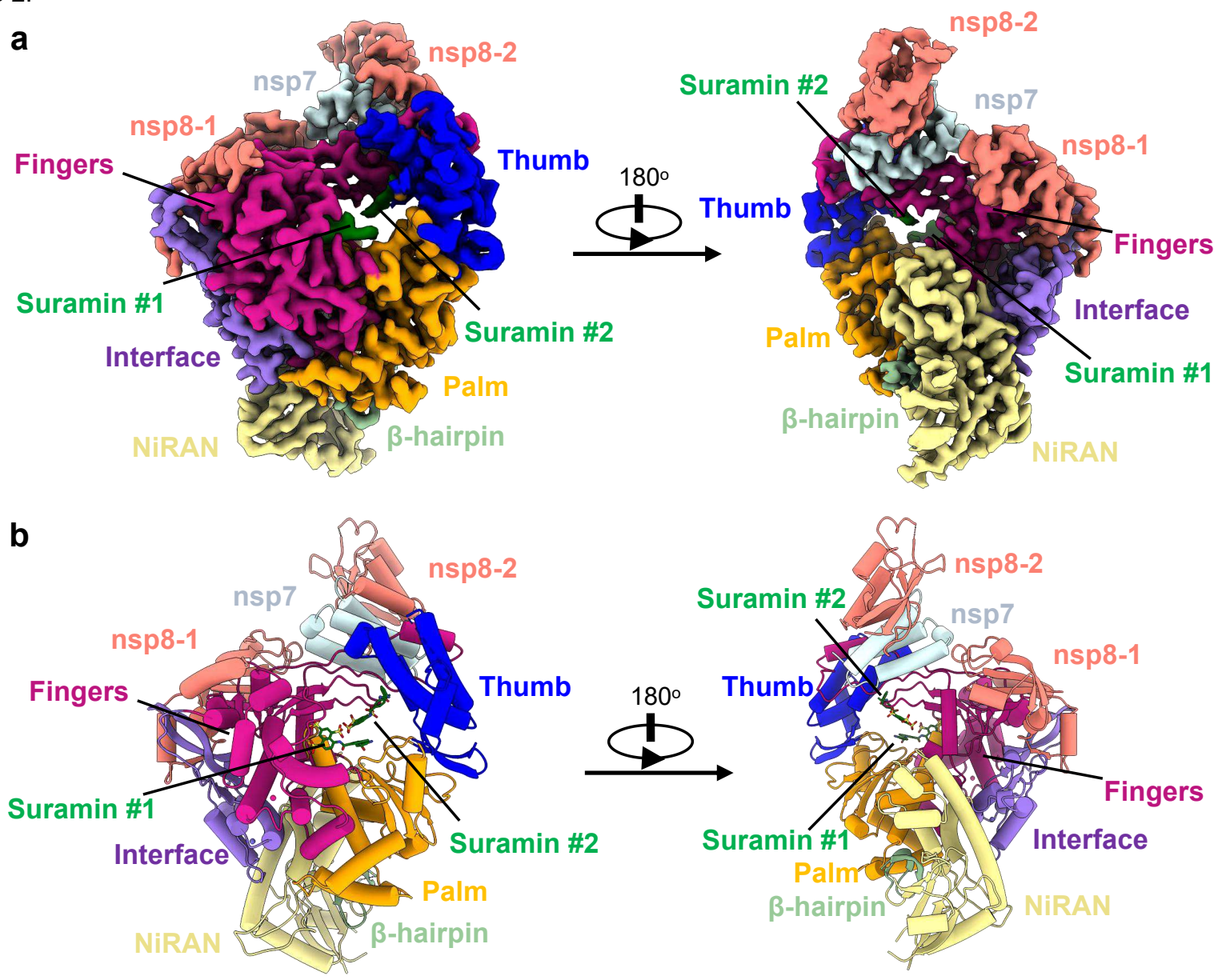


Figure 3.

a

nsp12(1-932)

$\downarrow$
$\downarrow$

365

$\downarrow$

RNA dependent RNA Polymerase

932

G $\mathbf{F}$

A

B C D E

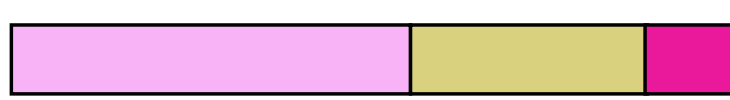

Fingers (S397-A581, K621-G679)

Motif G: 499-511

Motif C: $753-767$

Palm (T582-P620, T680-Q815)

Motif F: 544-560

Motif D: 771-796

Thumb (H816-E920)

Motif A: 612-626

Motif E: $\mathbf{8 1 0 - 8 2 0}$

Motif B: $678-710$

b

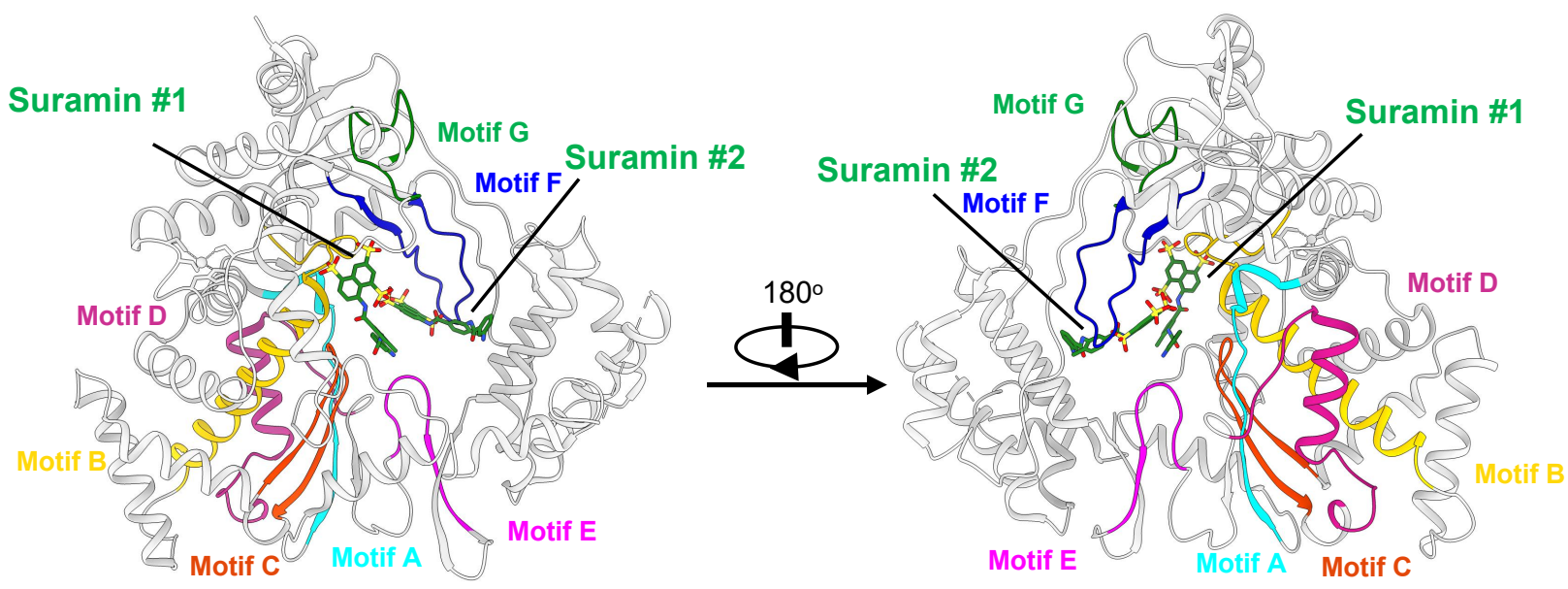


Figure 4.

a

C

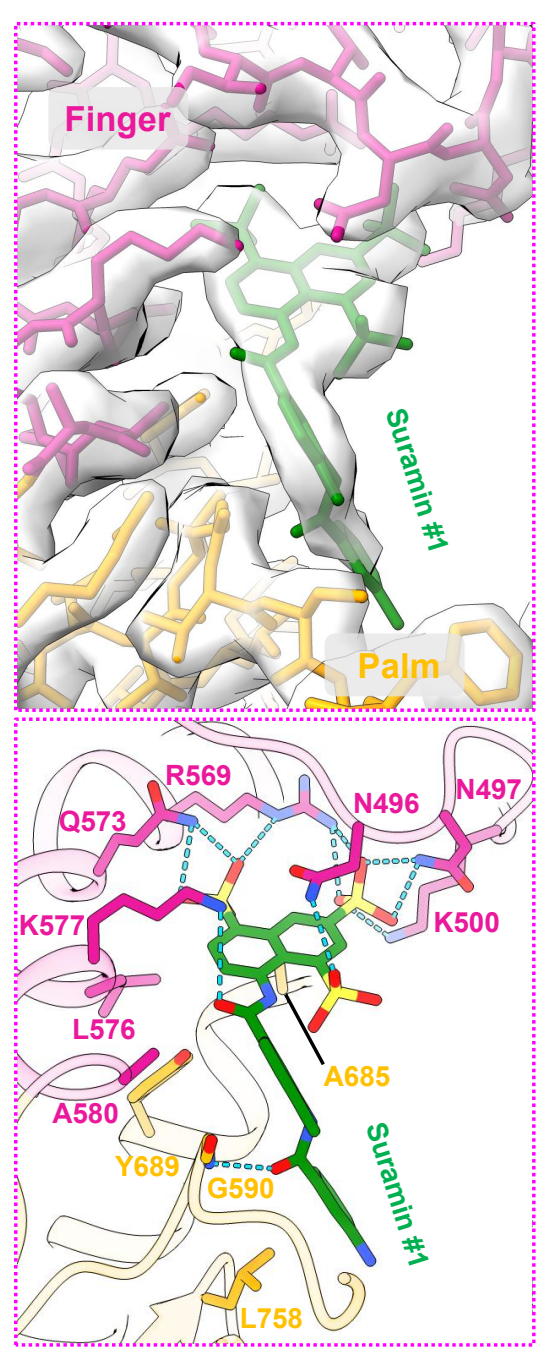

b

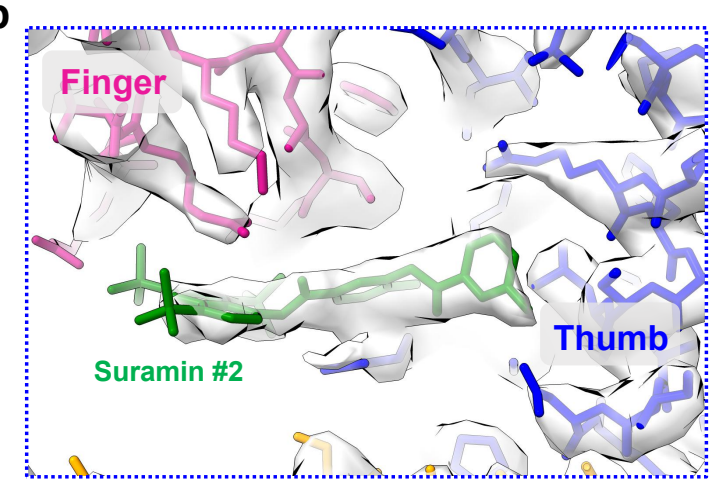

d

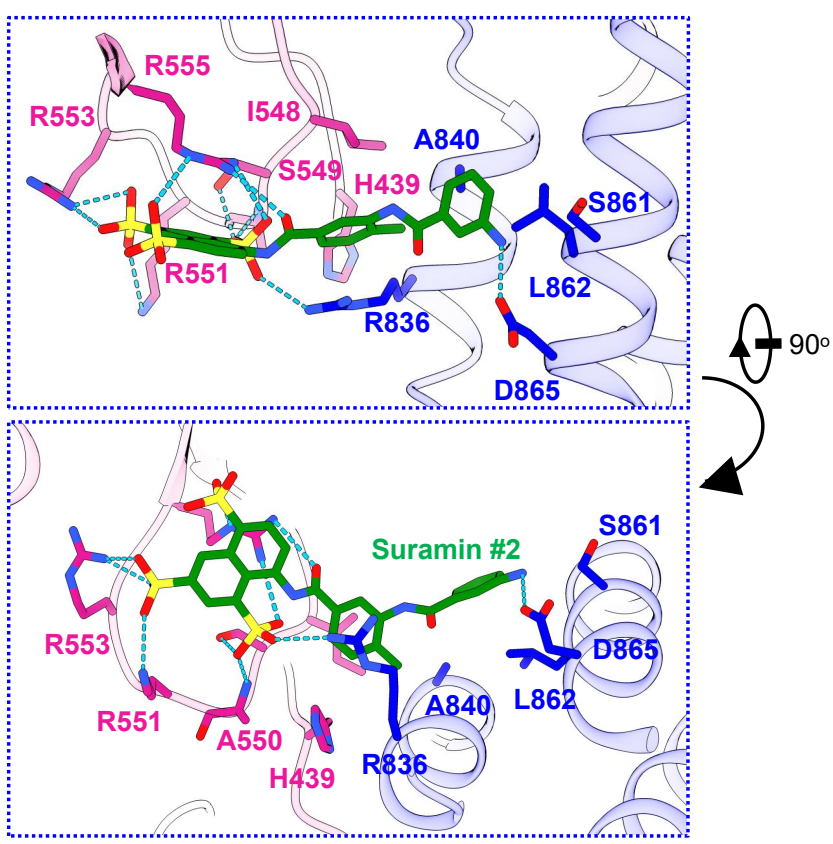


Figure 5.

a

RdRp complex (suramin bound)

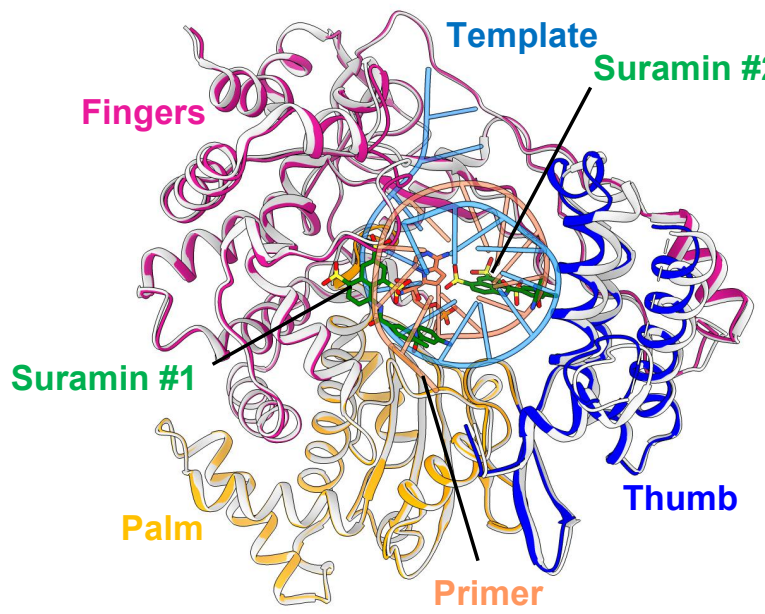

b

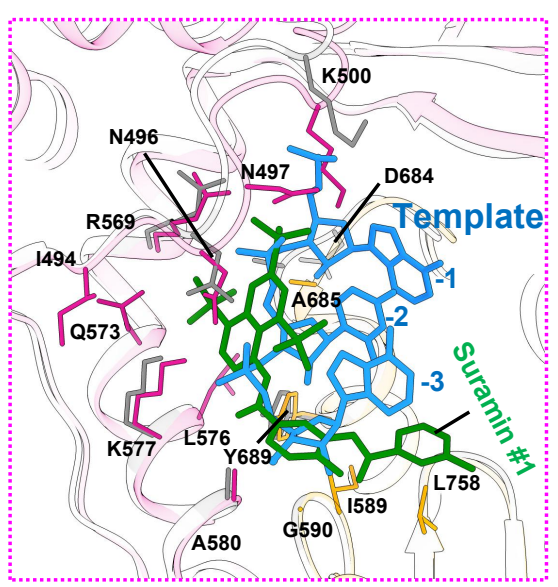

RdRp complex ( remdesivir bound)

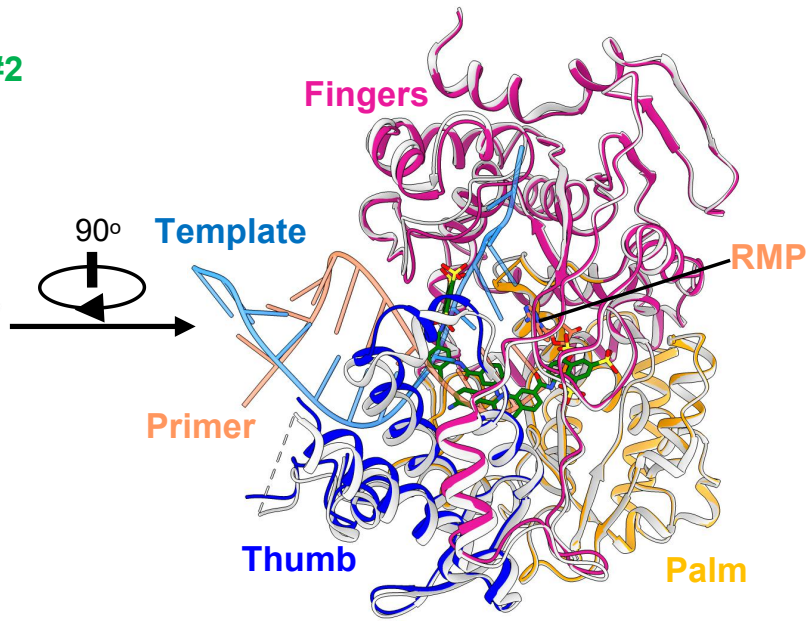

C

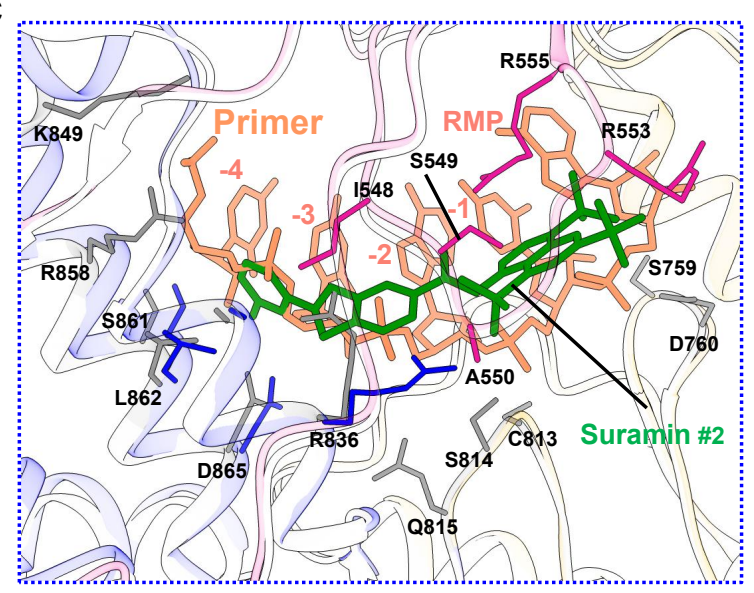


Figure 6.

a

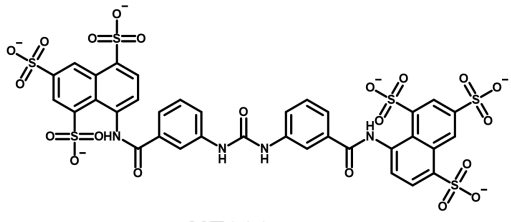

NF023

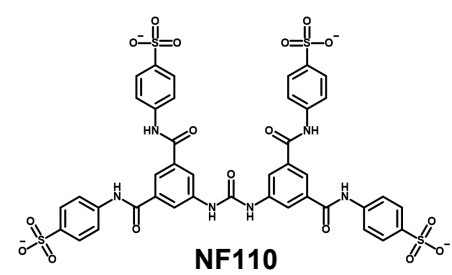

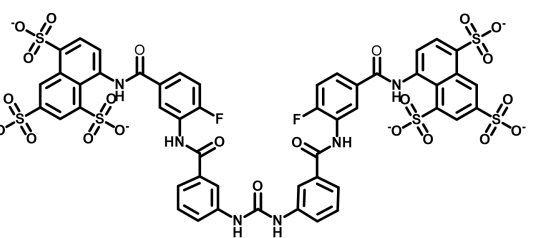
NF157

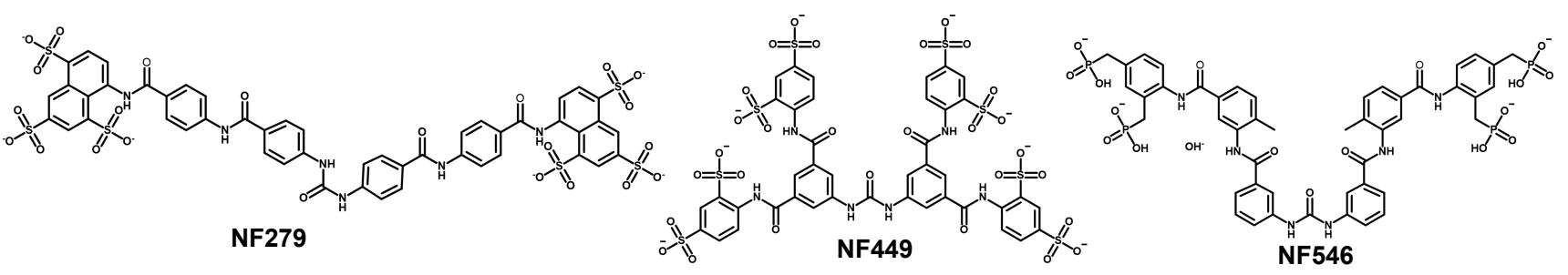

b
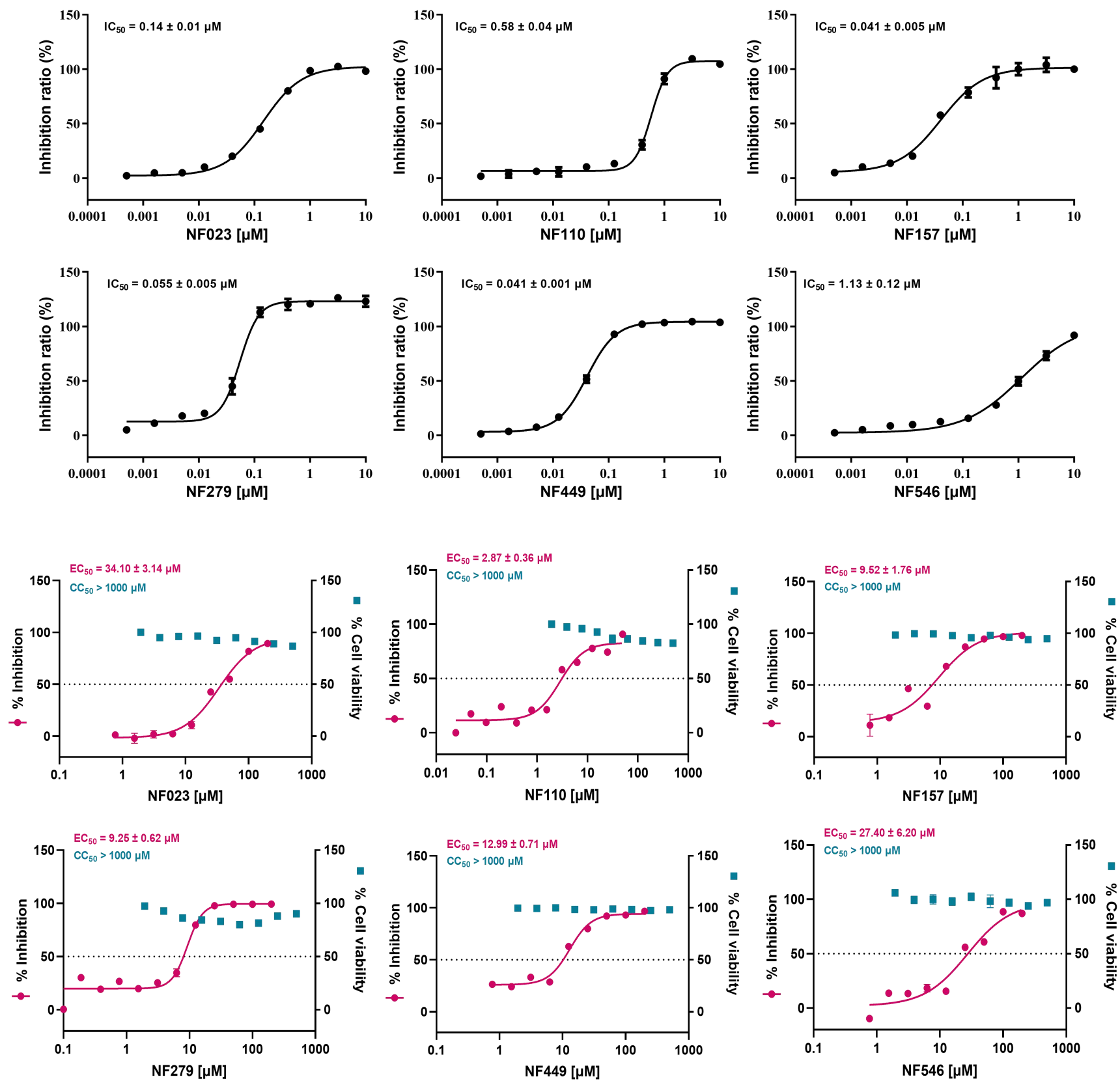
Figure S1

a

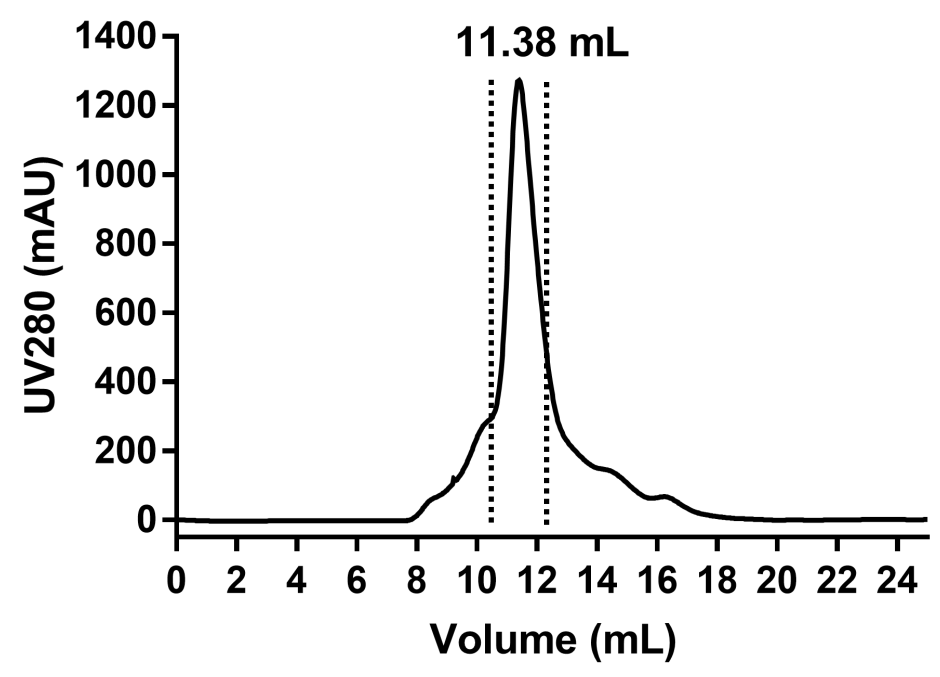

b

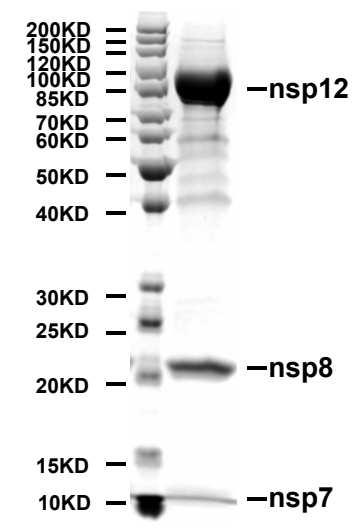

C

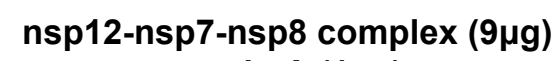
+ poly-A (1 $\mu \mathrm{g})$
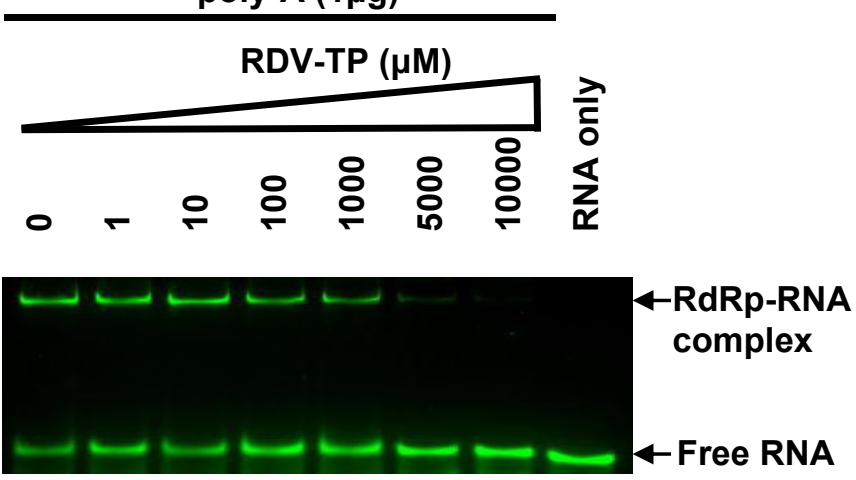

e

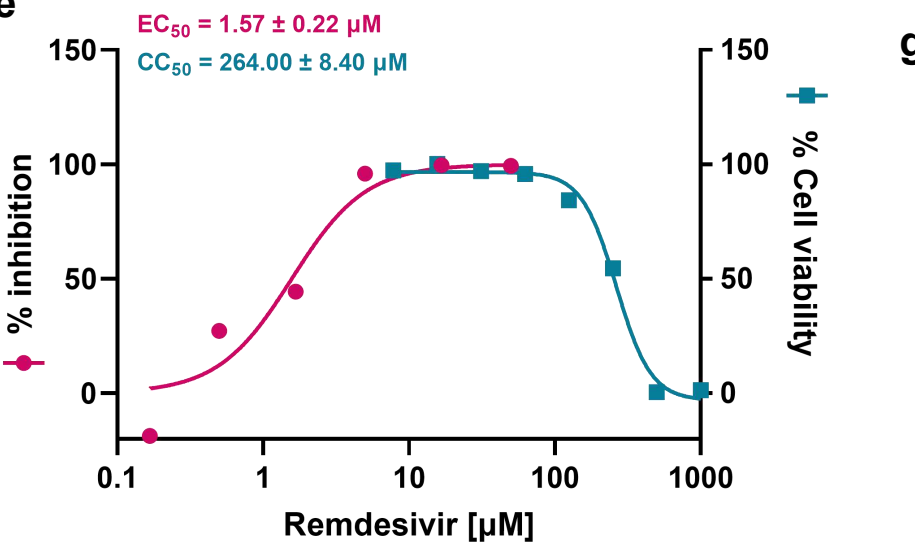

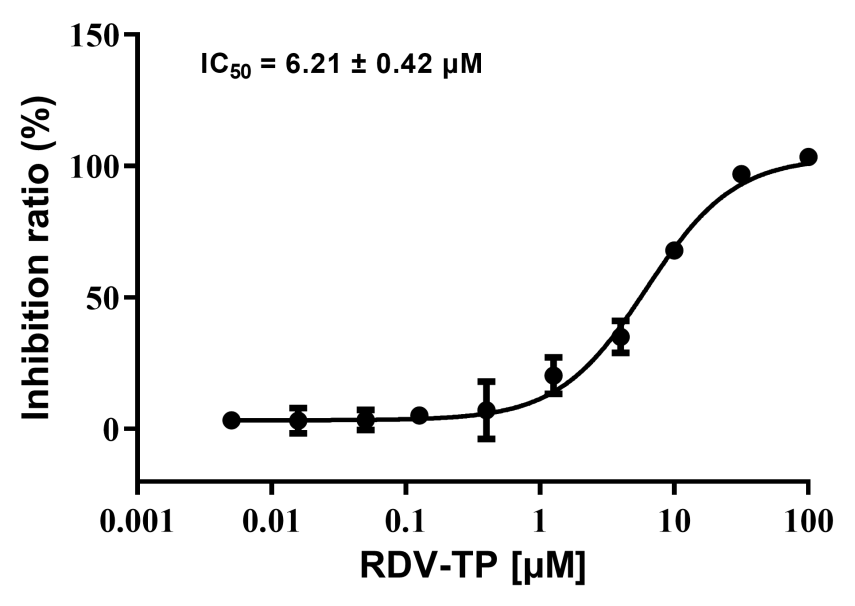

g

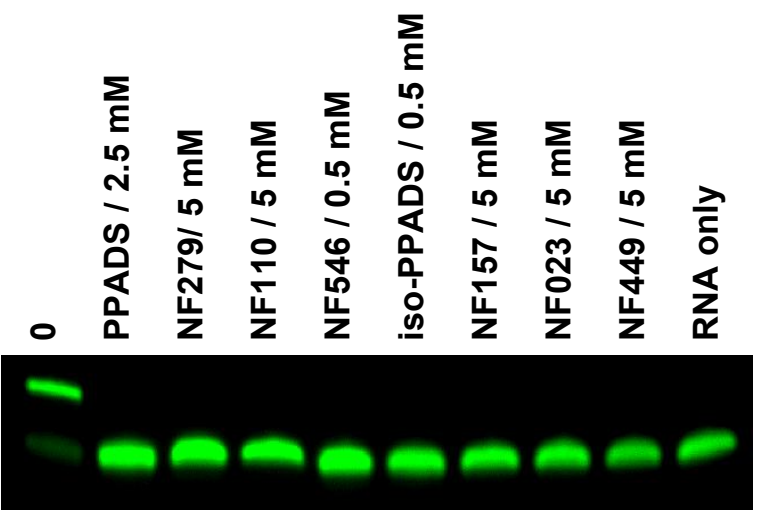

f

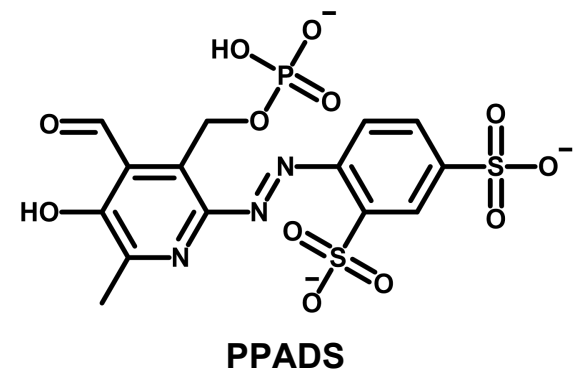

PPADS<smiles>Cc1nc(N=Nc2cc(S(=O)(=O)[O-])ccc2S(=O)(=O)[O-])c(COP(=O)([O-])O)c(C=O)c1O</smiles>

iso-PPADS 


\section{Figure $\mathbf{S 2}$}

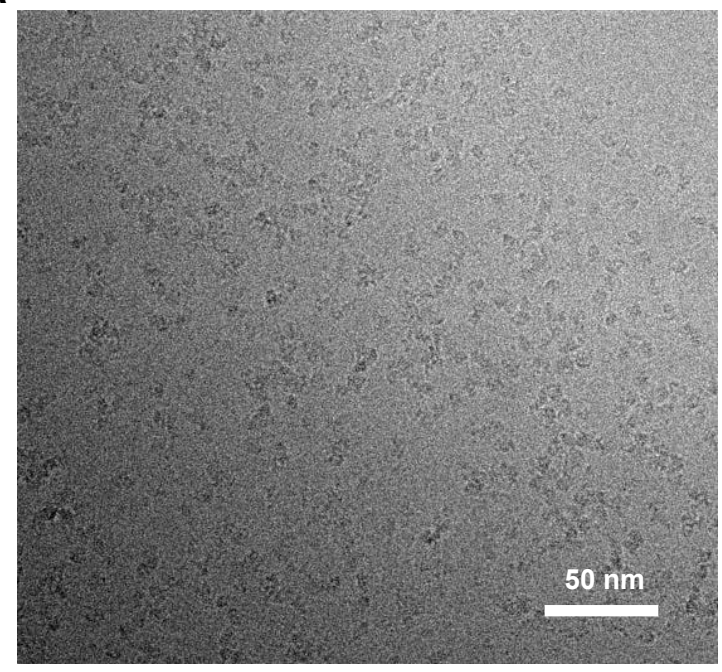

b

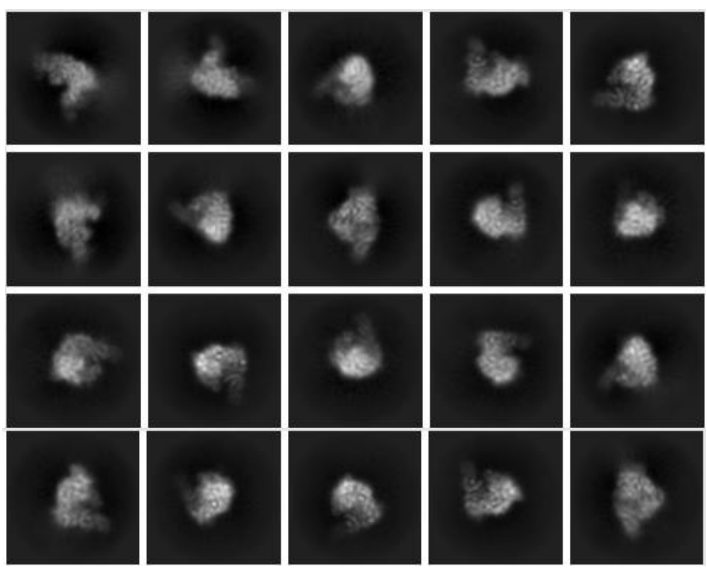

C

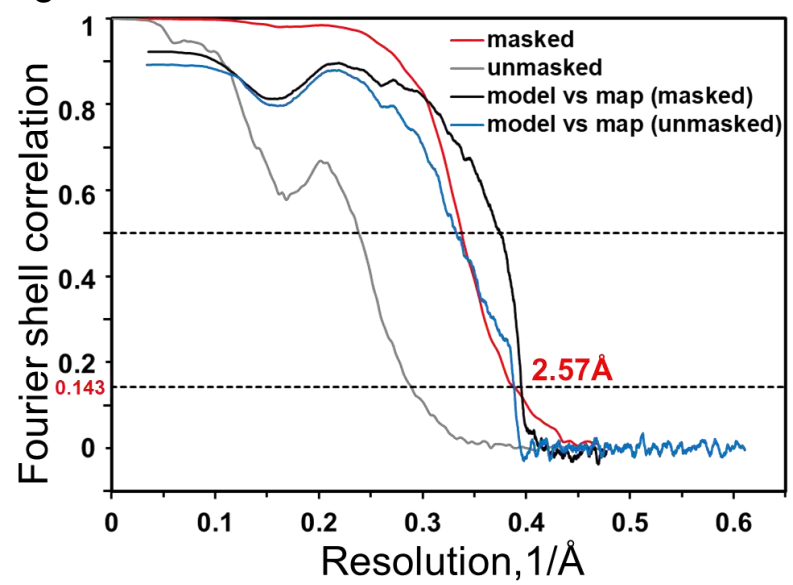

d

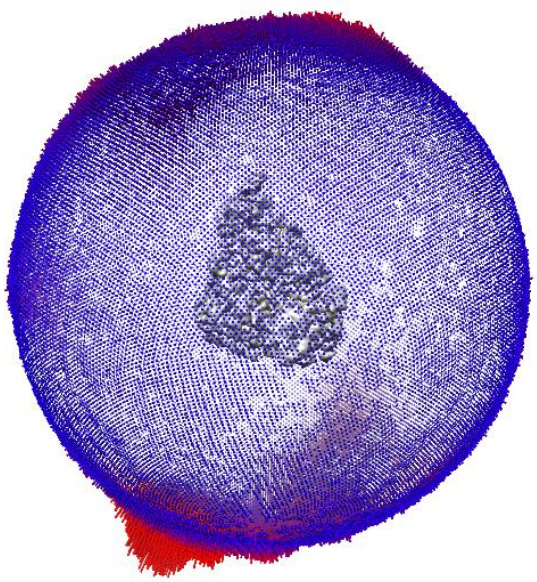

e

Motion correction

11,846

CTF estimation

10,241 Autopick micrographs $\longrightarrow$ microg,557,180 particles $\frac{\text { D }}{\mathrm{D}}$
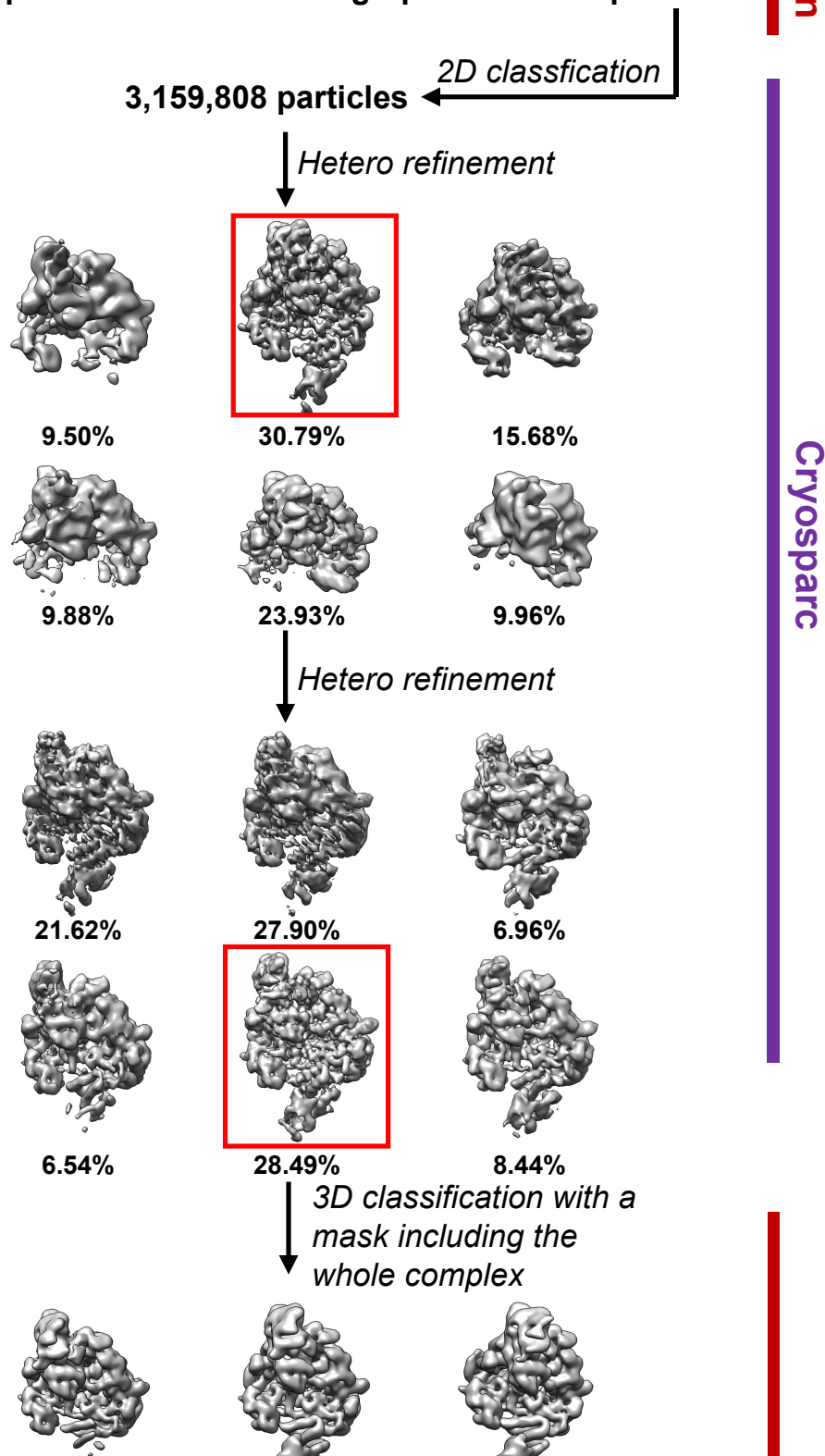
$\downarrow$ Hetero refinement

$\Omega$
$\frac{1}{2}$
$\frac{1}{0}$
$\frac{1}{3}$
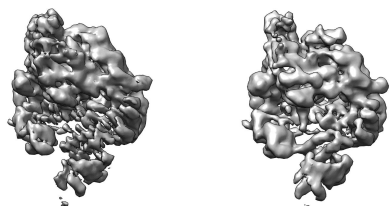

$27.90 \%$

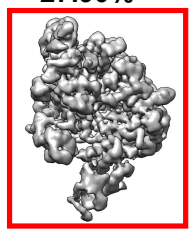

$6.96 \%$

$28.49 \%$

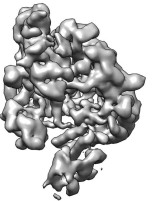

$8.44 \%$

$3 D$ classification with a mask including the whole complex

$21.34 \%$
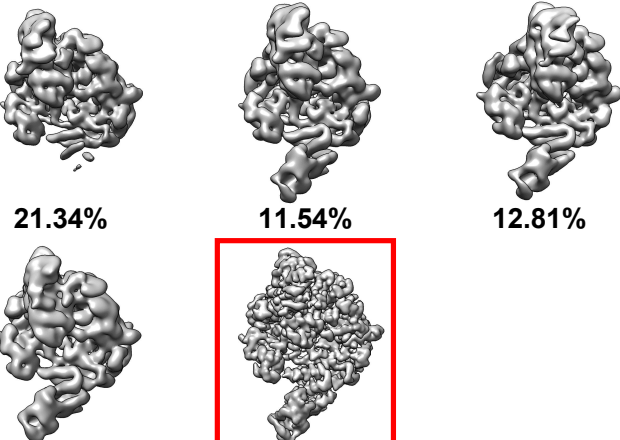

$19.75 \%$

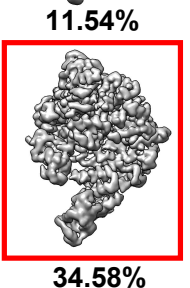

$12.81 \%$

$3 D$ refinement CTF refinement Bayesian polish
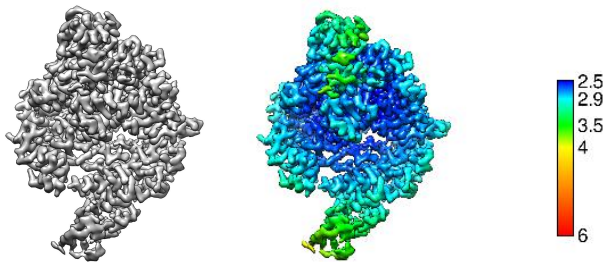

$2.57 \AA$

95,845 particles 
Figure S3

a

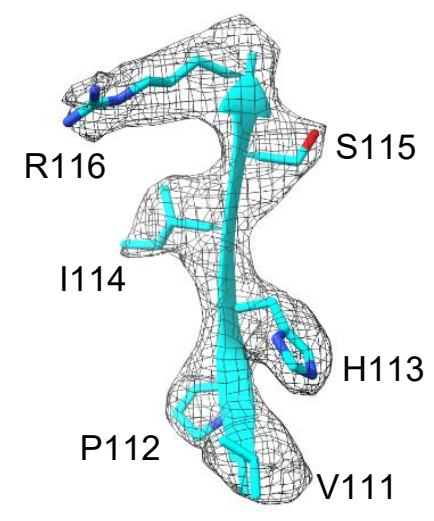

residues 111-116

(nsp12)
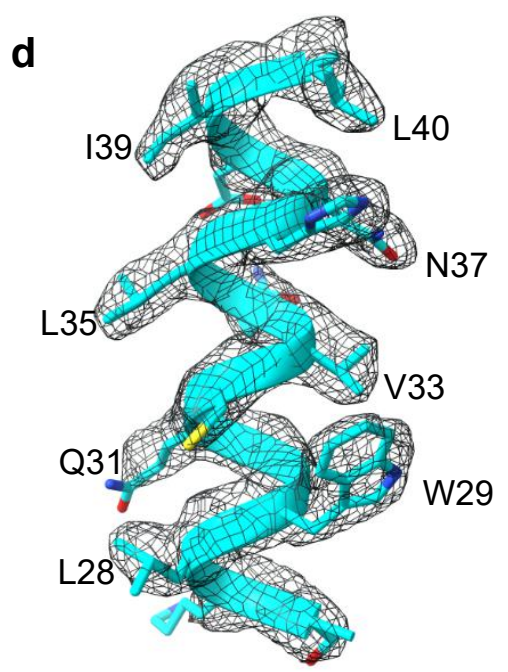

residues 26-40

(nsp7) b

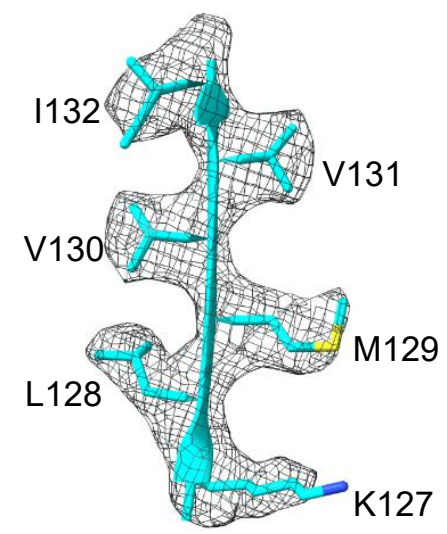

residues 127-132

(nsp8-1)

e

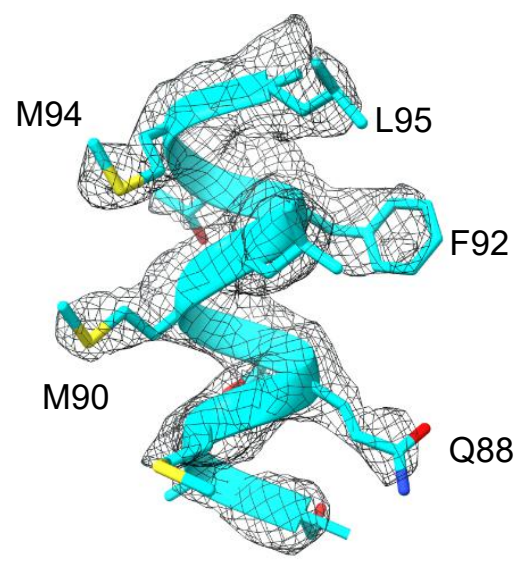

residues 85-95

(nsp8-2)
C

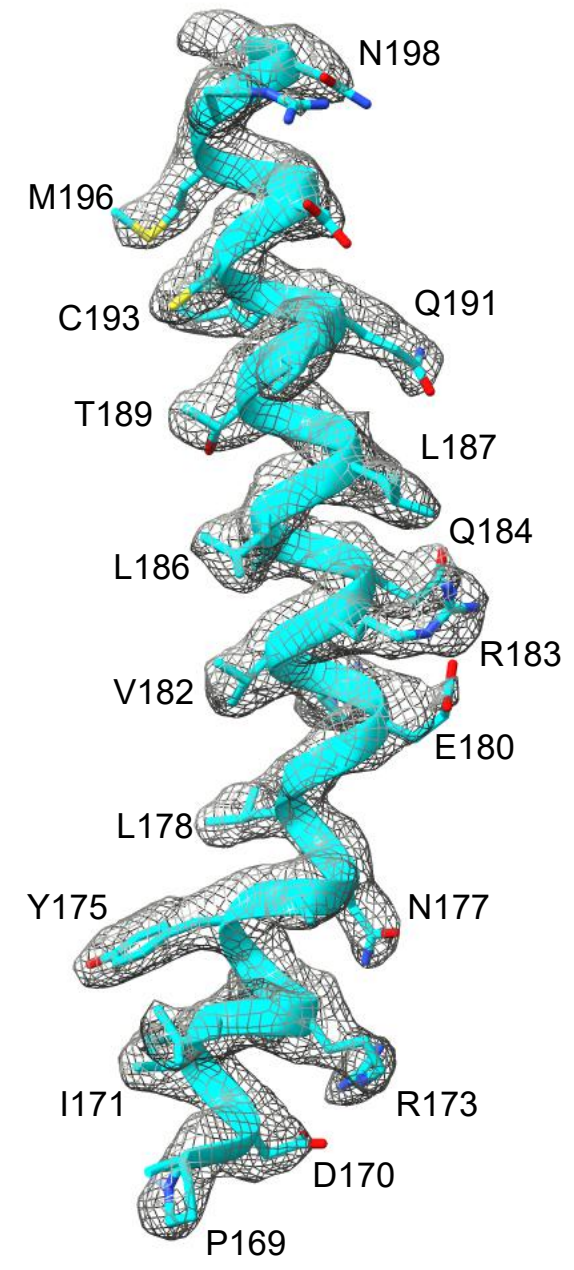

residues 169-199

(nsp12)

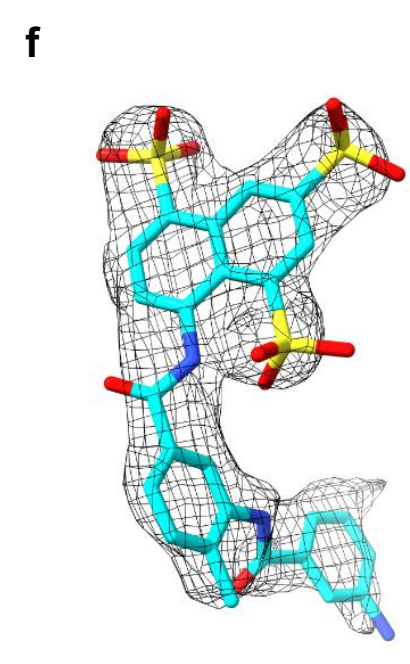

Suramin \#1 g

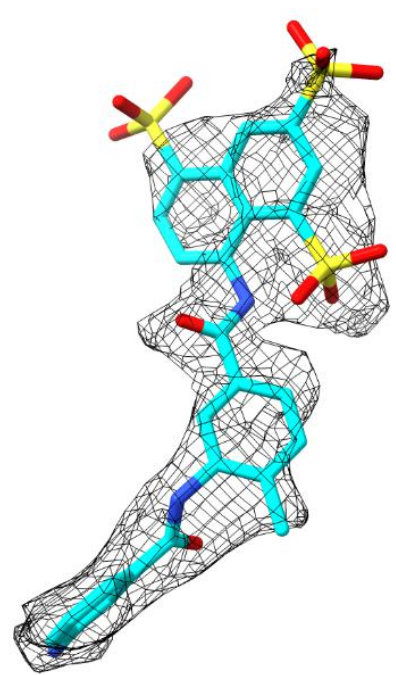

Suramin \#2 
Figure S4

RdRp complex (suramine bound)

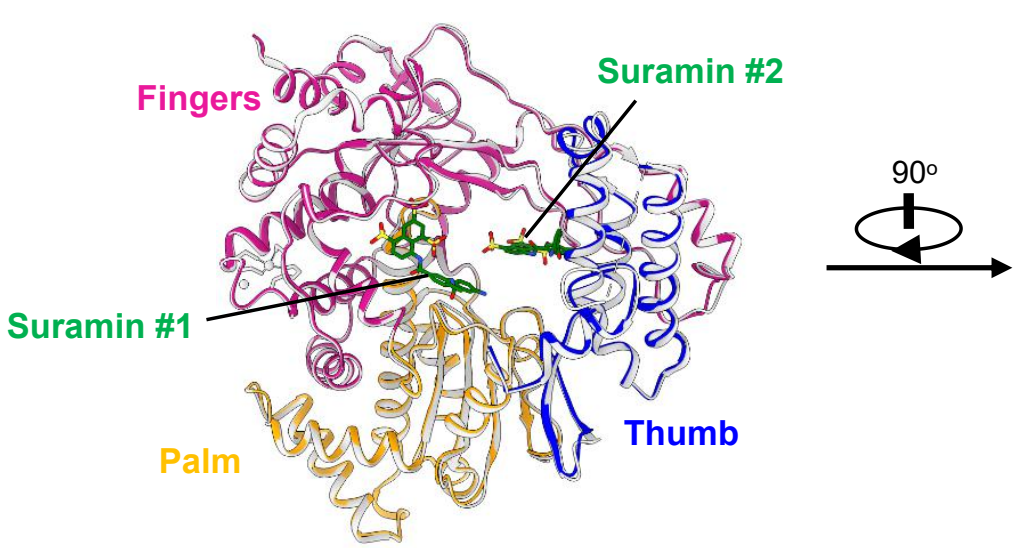

RdRp complex (Apo)

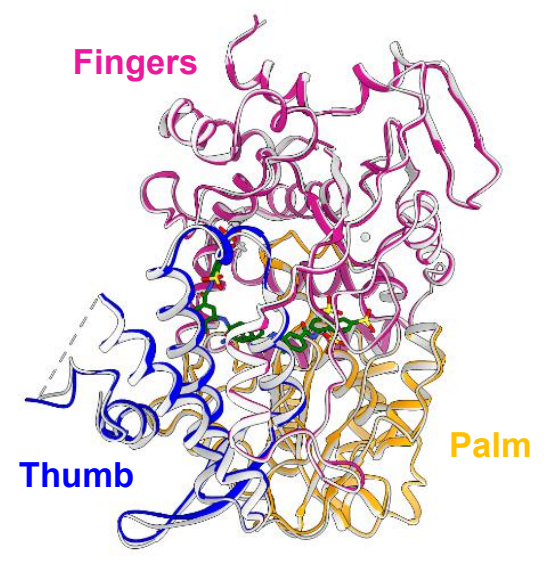


Figure S5
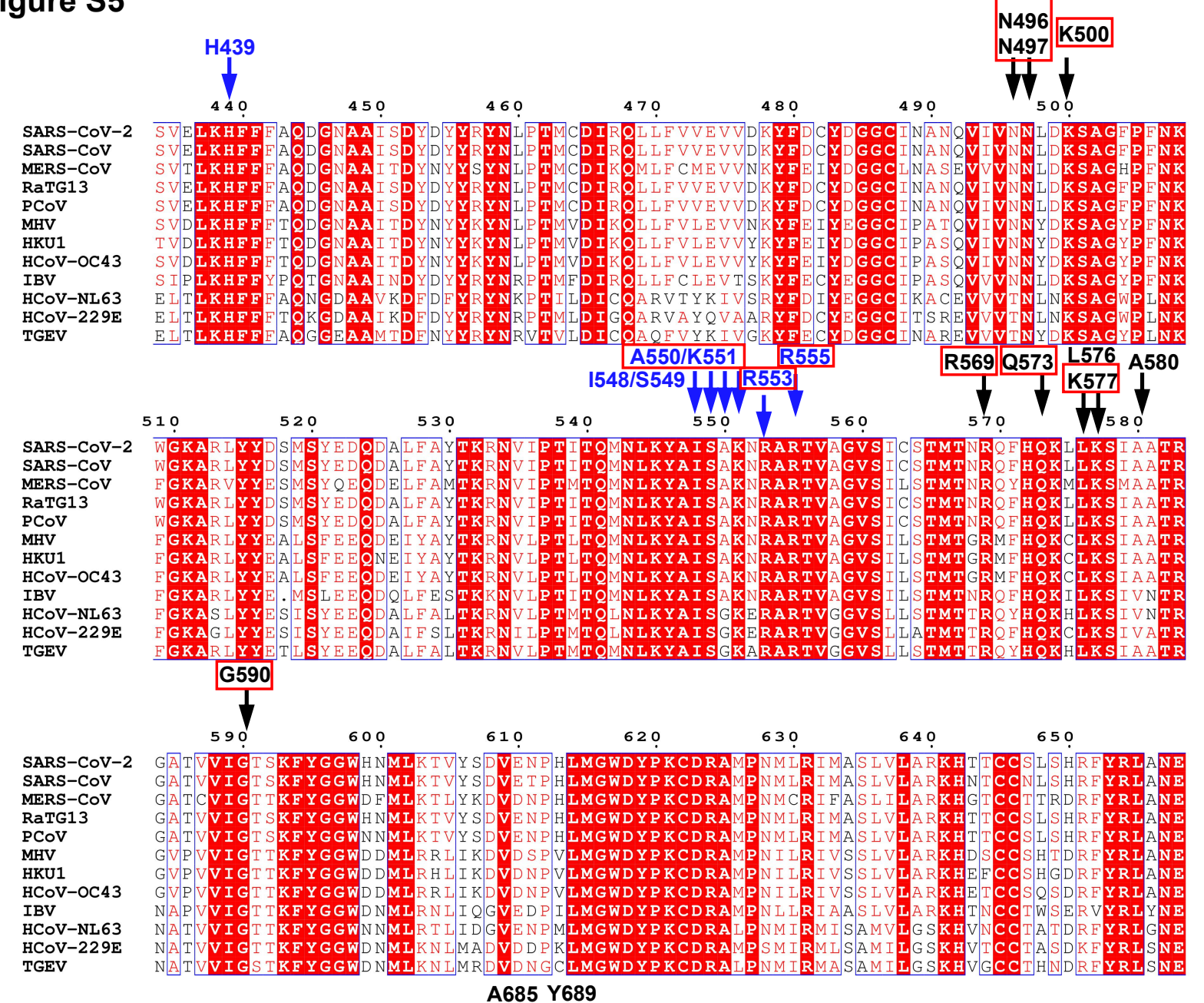

SARS-COV-2
SARS-COV
MERS-COV
RaTG13
PCOV
MHV
HKU1
HCOV-OC43
IBV
HCOV-NL63
HCOV-229E
TGEV

SARS-CoV-2
SARS-COV
MERS-CoV
RaTG13
PCOV
MHV
HKU1
HCOV-OC43
IBV
HCOV-NL63
HCOV-229E
TGEV

SARS-CoV-2
SARS-CoV
MERS-CoV
RaTG13
PCOV
MHV
HKU1
HCOV-OC43
IBV
HCOV-NL63
HCOV-229E
TGEV

IIII

$670 \quad 680 \quad 690$

700

710

720

730

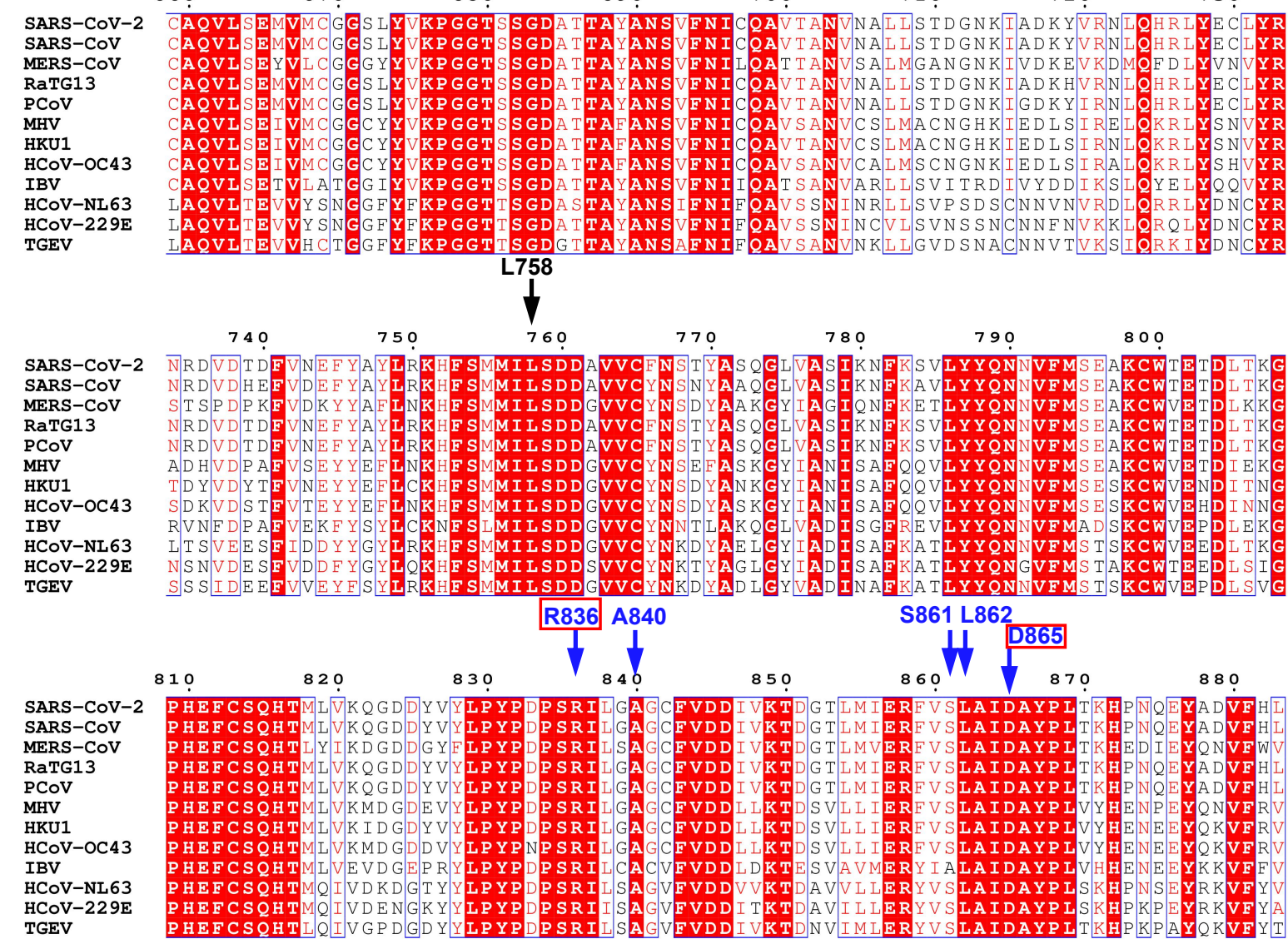

TGEV 


\section{Figure S6}

a

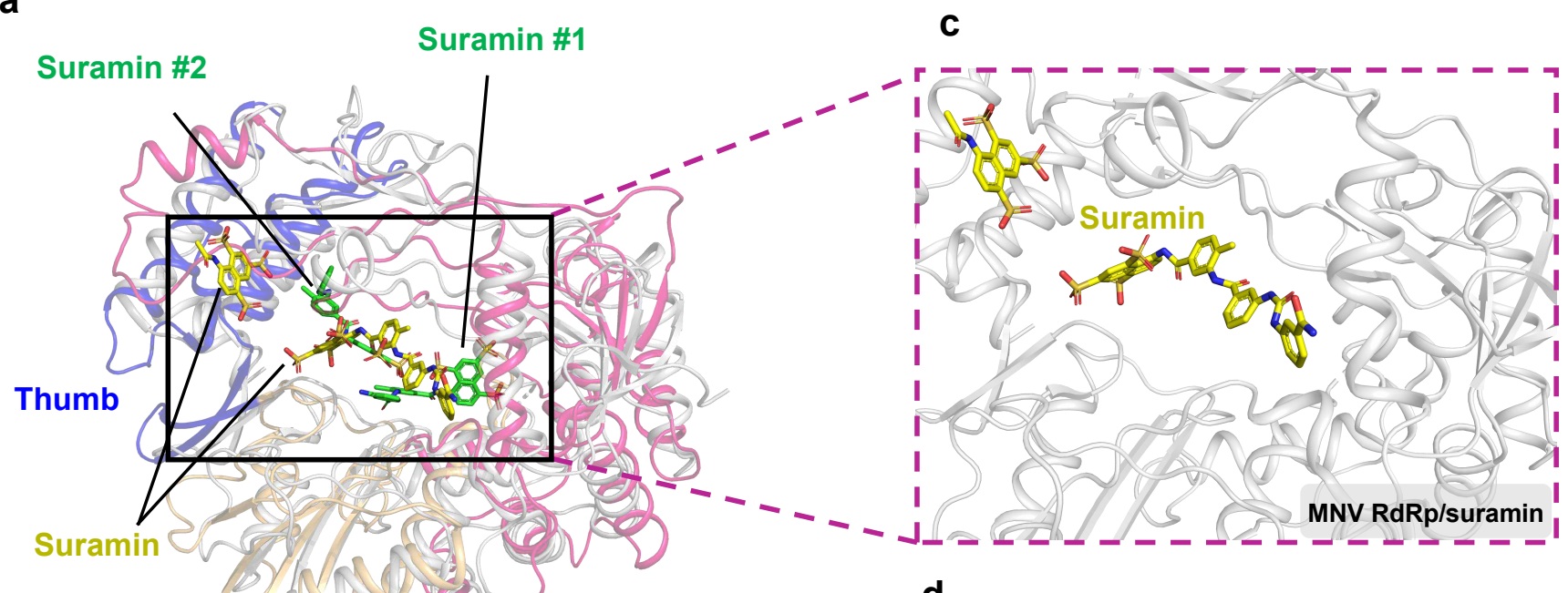

d

b
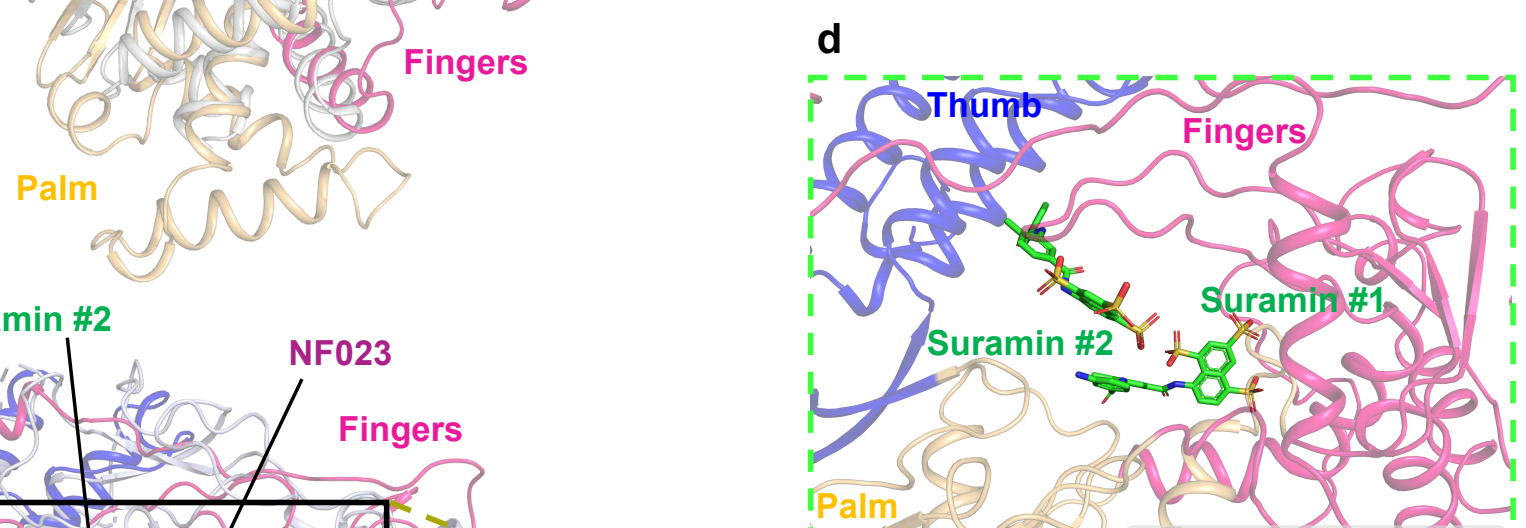

b Suramin \#2

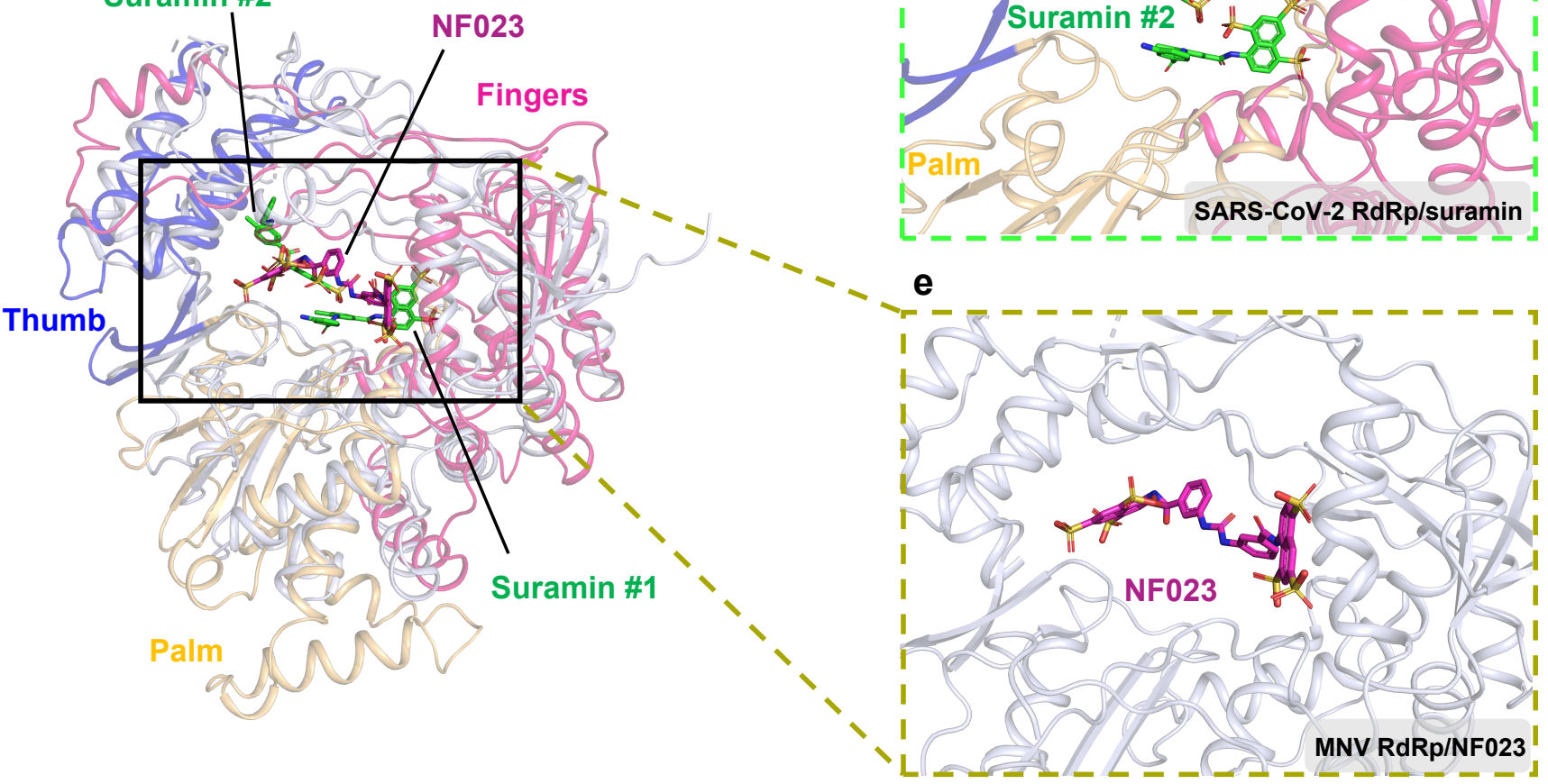


Figure S7

a

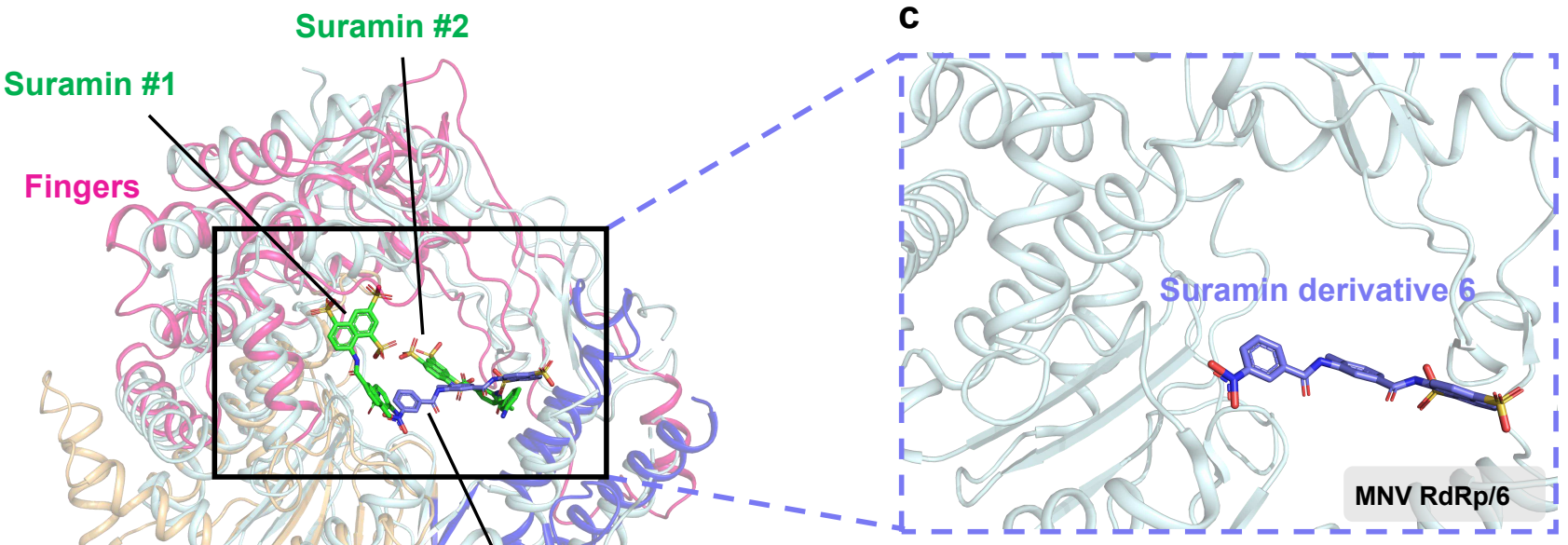

b suramin \#1

Suramin \#2

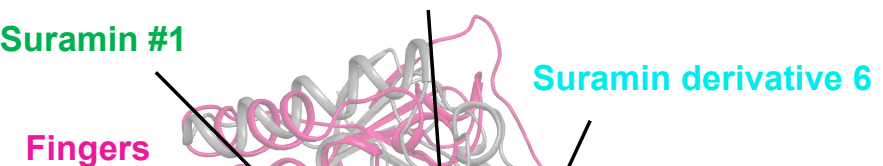

Finger

Palm
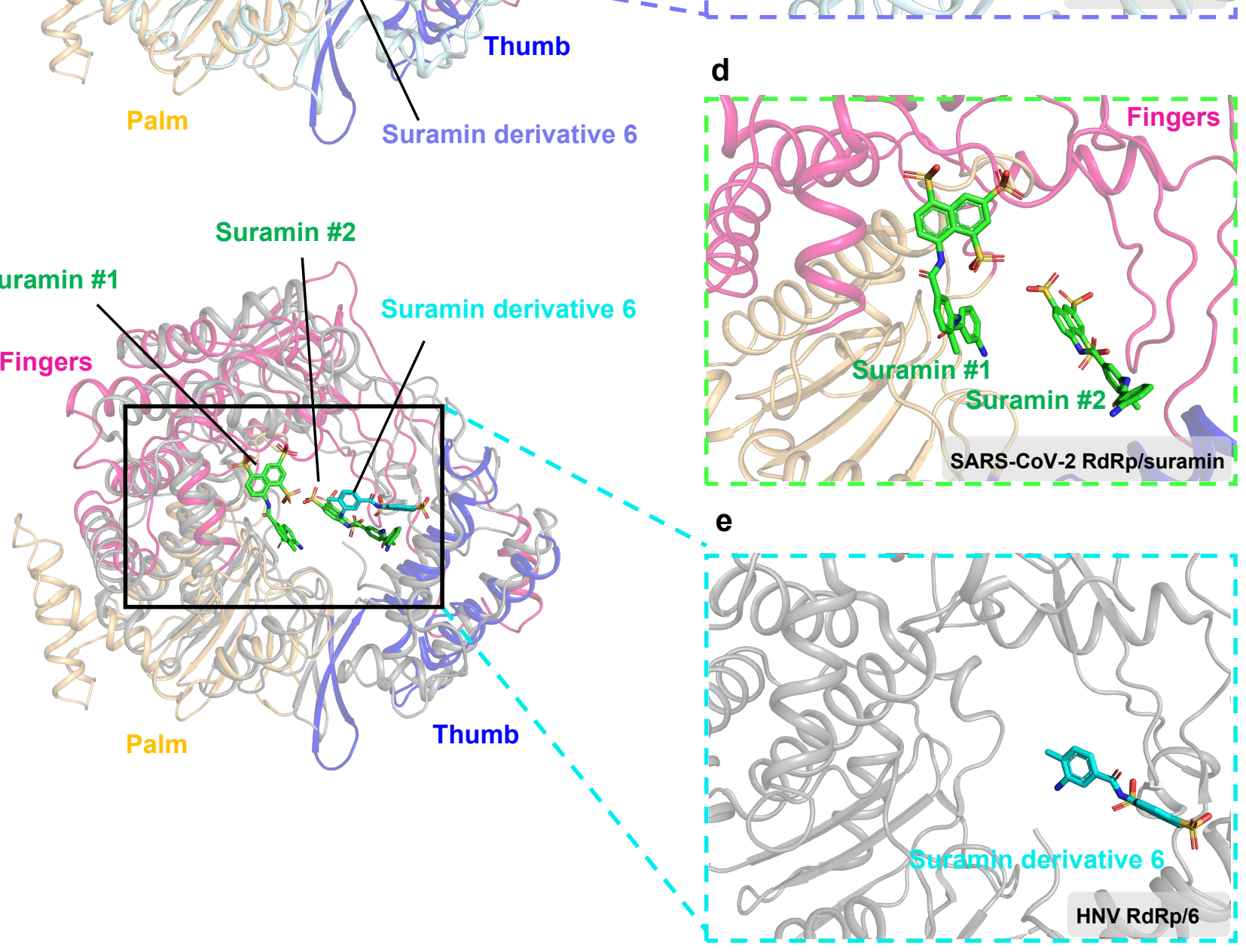
Extended Data Table 1. Cryo-EM data collection, refinement, and validation statistics

\begin{tabular}{|c|c|}
\hline & suramin \\
\hline \multicolumn{2}{|l|}{ Data collection and Processing (for each dataset) } \\
\hline Microscope & Titan Krios \\
\hline Voltage (keV) & 300 \\
\hline Camera & Gatan K3 ${ }^{\text {TM }}$ Camera \\
\hline Magnification & 46,773 \\
\hline Pixel size at detector ( $\AA /$ pixel $)$ & 1.069 \\
\hline Total electron exposure (e-/Å2) & 68 \\
\hline Exposure rate (e-/pixel/sec) & 26 \\
\hline Number of frames collected during exposure & 36 \\
\hline Defocus range $(\mu \mathrm{m})$ & -0.5 to -2.0 \\
\hline Phase plate (if used) & N/A \\
\hline -phase shift range (in degrees) & $\mathrm{N} / \mathrm{A}$ \\
\hline -number of images per phase plate position & N/A \\
\hline Automation software (EPU, SerialEM or manual) & SerialEM \\
\hline Tilt angle (if grid was tilted) & 0 \\
\hline Energy filter slit width (if used) & 20 \\
\hline Micrographs collected (no.) & 11,846 \\
\hline Micrographs used (no.) & 10,241 \\
\hline Total extracted particles (no.) & $8,557,180$ \\
\hline \multicolumn{2}{|l|}{ For each reconstruction: } \\
\hline Refined particles (no.) & 95,845 \\
\hline Final particles (no.) & 95,845 \\
\hline Point-group or helical symmetry parameters & C1 \\
\hline \multicolumn{2}{|l|}{ Resolution (global, $\AA$ ) } \\
\hline FSC 0.5 (unmasked/masked) & $4.2 / 3.5$ \\
\hline FSC 0.143 (unmasked/masked) & $3.0 / 2.6$ \\
\hline Resolution range (local, $\AA$ ) & $2.5-6.0$ \\
\hline Map sharpening B factor $(\AA 2)$ & -27.866 \\
\hline \multicolumn{2}{|l|}{ Model composition (for each model) } \\
\hline Protein & 1181 \\
\hline Ligands & 4 \\
\hline RNA/DNA & 0 \\
\hline \multicolumn{2}{|l|}{ Model Refinement (for each model) } \\
\hline Refinement package & PHENIX-1.14_3260 \\
\hline -real or reciprocal space & real space \\
\hline Initial model used (PDB code) & 7BV1 \\
\hline Model-Map CC & 0.85 \\
\hline Model resolution $(\AA)$ & 2.7 \\
\hline FSC threshold & 0.5 \\
\hline \multicolumn{2}{|l|}{ B factors $(\AA 2)$} \\
\hline Protein residues & 63.49 \\
\hline Ligands & 64.64 \\
\hline RNA/DNA & N/A \\
\hline \multicolumn{2}{|l|}{ R.m.s. deviations from ideal values } \\
\hline Bond lengths $(\AA)$ & 0.012 \\
\hline Bond angles $\left({ }^{\circ}\right)$ & 0.904 \\
\hline \multicolumn{2}{|l|}{ Validation (for each model) } \\
\hline MolProbity score & 1.34 \\
\hline CaBLAM outliers & 0.96 \\
\hline Clashscore & 5.43 \\
\hline Poor rotamers (\%) & 0.76 \\
\hline C-beta deviations & 0.00 \\
\hline EMRinger score (if better than $4 \AA$ resolution) & 3.63 \\
\hline \multicolumn{2}{|l|}{ Ramachandran plot } \\
\hline Favored $(\%)$ & 97.77 \\
\hline Outliers (\%) & 0.00 \\
\hline
\end{tabular}


Extended Data Table 2. Interactions between suramin and nsp12 in the RdRp-suramin structure.

\begin{tabular}{|c|c|c|c|c|c|}
\hline & suramin & interation & distance $(\AA)$ & & nsp12 \\
\hline \multirow{12}{*}{ suramin \#1 } & sulfonate1 & hydrogen bond & 3.2 & side chain & N496 \\
\hline & \multirow{4}{*}{ sulfonate3 } & hydrogen bond & 2.5 & side chain & N497 \\
\hline & & hydrogen bond & 3.9 & main chain & N497 \\
\hline & & hydrogen bond & 3.2 & side chain & $\mathrm{K} 500$ \\
\hline & & hydrogen bond & 2.8 & side chain & R569 \\
\hline & \multirow{3}{*}{ sulfonate5 } & hydrogen bond & 3.1 & side chain & R569 \\
\hline & & hydrogen bond & 2.8 & side chain & Q573 \\
\hline & & hydrogen bond & 3.4 & side chain & Q573 \\
\hline & B-C linker & hydrogen bond & 3.6 & side chain & K577 \\
\hline & naphthalene ring & cation- $\pi$ stacking & & side chain & K577 \\
\hline & \multirow[t]{2}{*}{ C-D linker } & hydrogen bond & 2.9 & main chain & G590 \\
\hline & & van der Waals contact & & & L576, A580, A685, Y689, 1758 \\
\hline \multirow{12}{*}{ suramin \#2 } & \multirow{4}{*}{ sulfonate1 } & hydrogen bond & 2.6 & main chain & A550 \\
\hline & & hydrogen bond & 3.7 & main chain & K551 \\
\hline & & hydrogen bond & 3.9 & side chain & R555 \\
\hline & & hydrogen bond & 3.2 & side chain & R836 \\
\hline & \multirow{3}{*}{ sulfonate3 } & hydrogen bond & 3.9 & side chain & K551 \\
\hline & & hydrogen bond & 2.1 & side chain & R553 \\
\hline & & hydrogen bond & 3.6 & side chain & R553 \\
\hline & sulfonate5 & hydrogen bond & 3.7 & side chain & R555 \\
\hline & B-C linker & hydrogen bond & 3.6 & side chain & R555 \\
\hline & ring $\mathrm{C}$ & cation- $\pi$ stacking & & side chain & R836 \\
\hline & ring $D$ & hydrogen bond & 3.0 & side chain & D865 \\
\hline & & van der Waals contact & & & H439, I548, S549, A840, S861, L862 \\
\hline
\end{tabular}




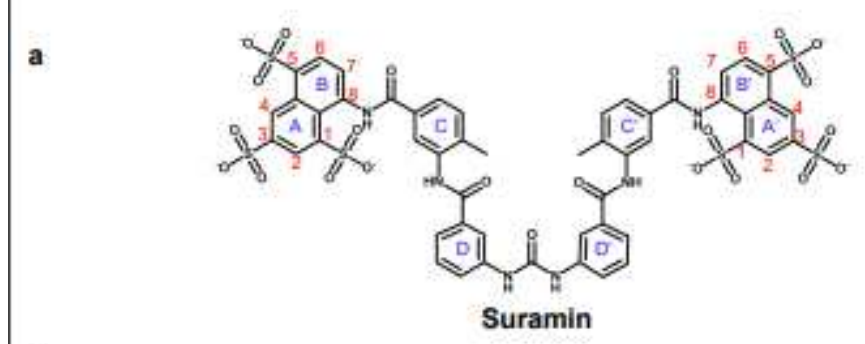

b

poly-A: SFAM-GCUAUGUGAGAUUAAGUUAU 3

3.
CoAUACACUCUAAUUCAAUAAAAAAAAAAA

poly-U: S'FAM-GCUAUGUGAGAUUAAGUUAU S

3. 11111111111111111111111
CGAUACACUCUAAUUCAAUAUUUUUU⿴囗十

c

d

nsp12-nsp7-nsp8 complex (9ug)

poly-A + $10 \mathrm{mM}$ UTP
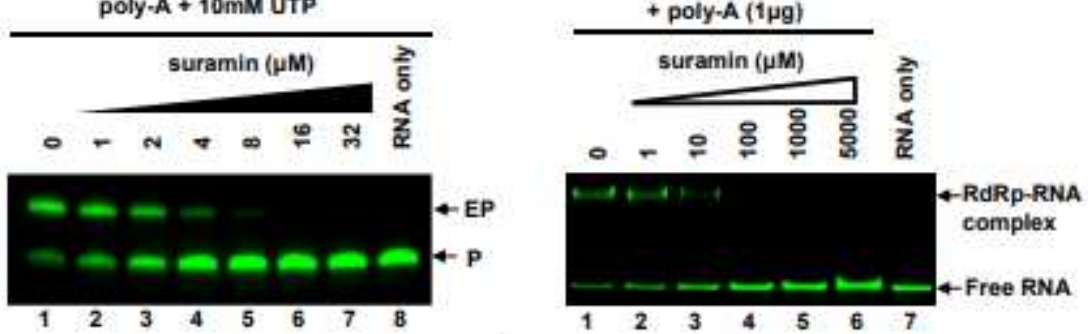

e

f
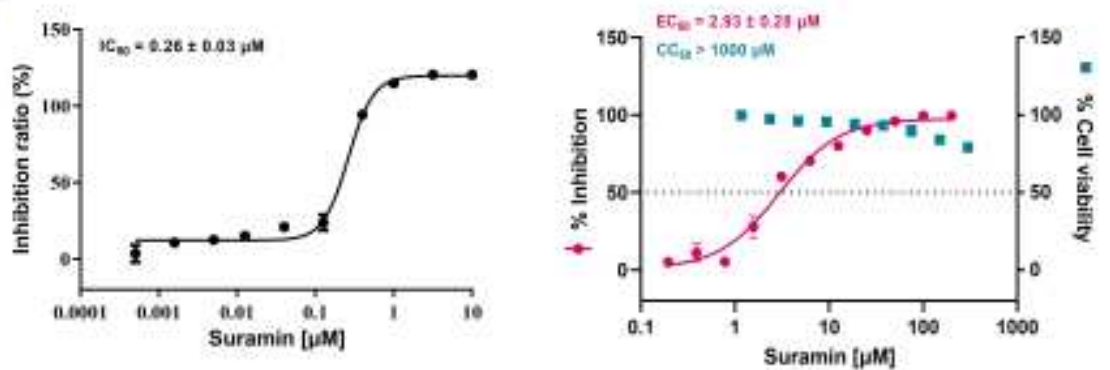

Figure 1

Inhibition of RdRp by suramin and the potential anti-SARS-CoV-2 effect of suramin a. The chemical structure of suramin. b. The 30-base template and 20-base primer duplex RNAs with a FAM at the $5^{\prime}$ of the primer. Poly-A was used in the gel-based elongation assay, while Poly-U was used in the fluorescencebased activity assay for SARS-CoV-2 RdRp. c. Elongation of partial RNA duplex by the purified RdRp complex and its inhibition by suramin. The EP means the elongated product, while the P means the primer RNA strand. d. Gel mobility shift of the RdRp-RNA complex and the effect of suramin. e. IC50 of suramin for RdRp complex. f. EC50 of suramin for SARS-CoV-2 inhibition and CC50 of suramin for cellbased toxicity. 


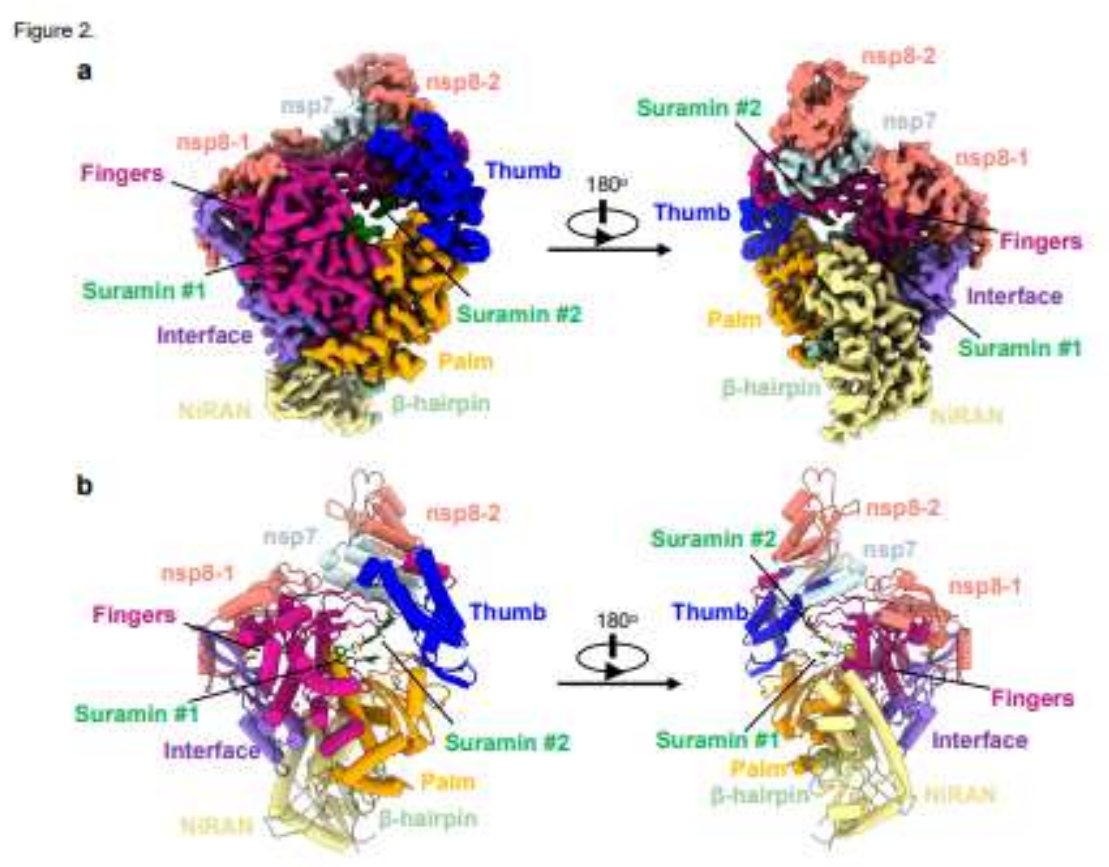

\section{Figure 2}

Overall Structures of the RdRp-suramin complex a. Two views of cryo-EM density map of the RdRpsuramin complex, SARS-CoV2 nsp12 contains a N-terminal extension composed of the $\beta$-hairpin (green), the NiRAN domain (yellow) and an interface domain (purple) adjacent to the RdRp core domain, which contains subdomains: fingers, palm, and thumb, colored with violet, orange, and blue, respectively. The nsp12 binds to a heterodimer of nsp7 (light green) and nsp8 (nsp8-2, red) as well as to a second subunit of nsp8 (nsp-1, red). The two bound suramin molecules are set as dark green. Color code is used throughout. b. Two views of the overall structure of the RdRp-suramin complex, the color scheme is according as above. 
a

nsp12(1-832)

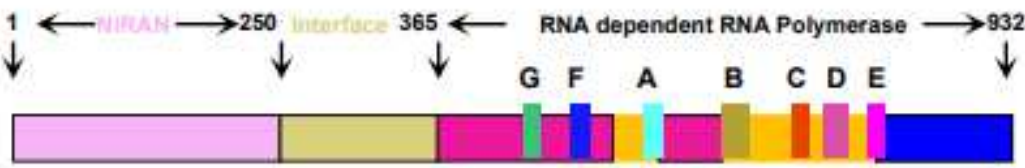

Fingers (\$397-A581, K621-G679)

Motif 6: 499.511 Motif C: 753-767

(T582-P620, T680-Q815)

Motif F: $544-560$

Motif D: $771-796$

Thumb (H816-E920)

Guif Acc 62 Motif E: $810-820$

Matif B: $578-710$

b

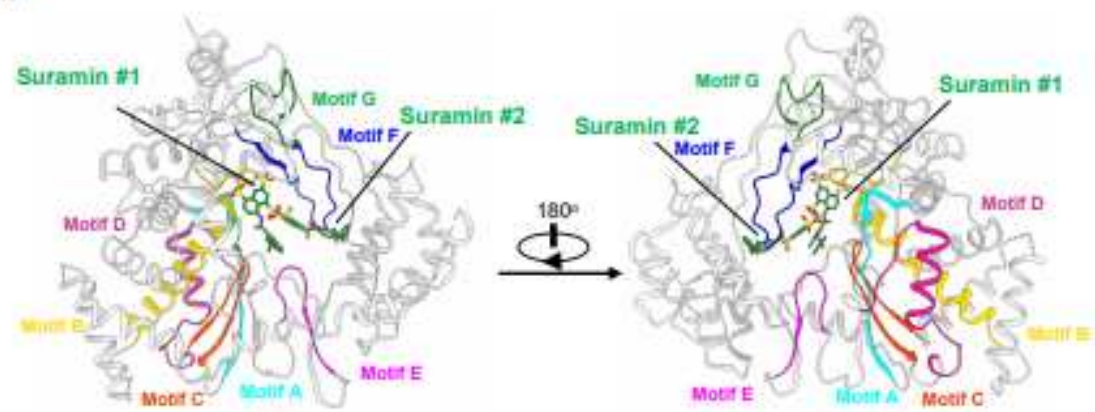

Figure 3

The conserved motif A-G in the RdRp-suramin complex. a. Schematic diagram for the components of the RdRp complex subunit nsp12, the motif ( $A$ to $G$ ) are highlighted. $b$. The color code is as follows: motif $A$ (cyan), motif $B$ (gold), motif $C$ (orange red), motif $D$ (medium violet red), motif $E$ (magenta), motif $F$ (blue), and motif $\mathrm{G}$ (green).

a

c

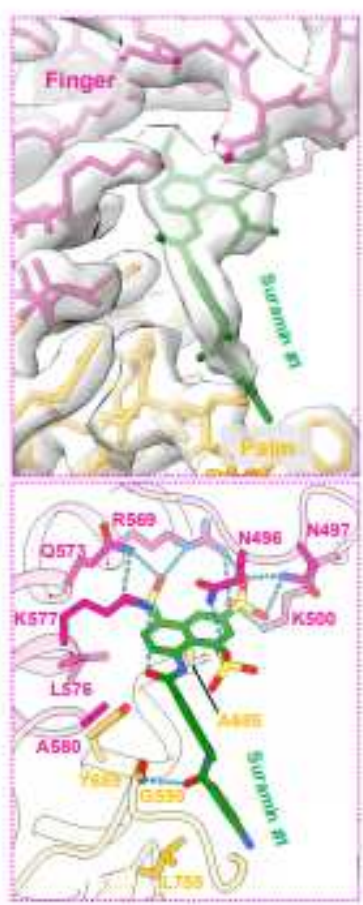

b
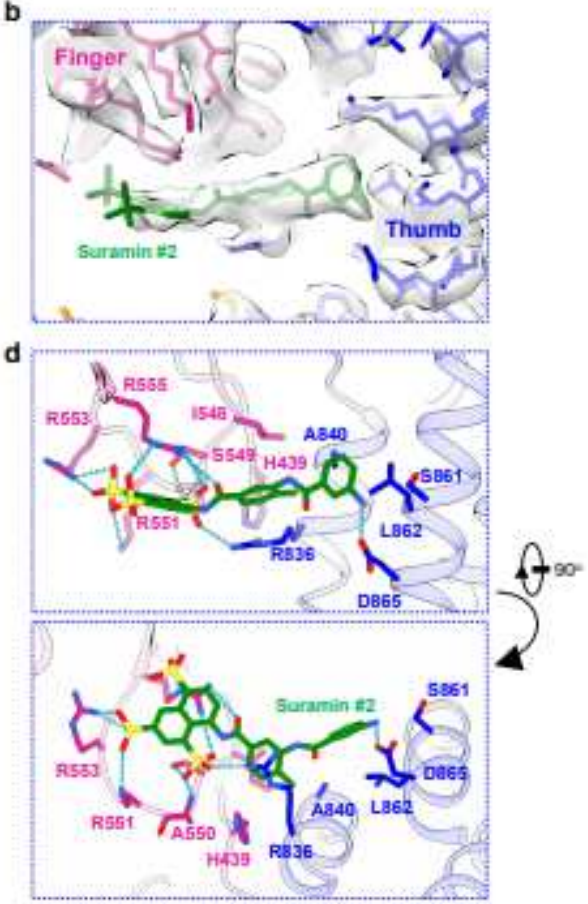

Figure 4 
Close views of the interactions between the SARS-CoV-2 RdRp and suramin a-b. The maps for the two suramin molecules. c-d. Interactions of the two suramin molecules with RdRp. The hydrogen bond is displayed as green dashed line.

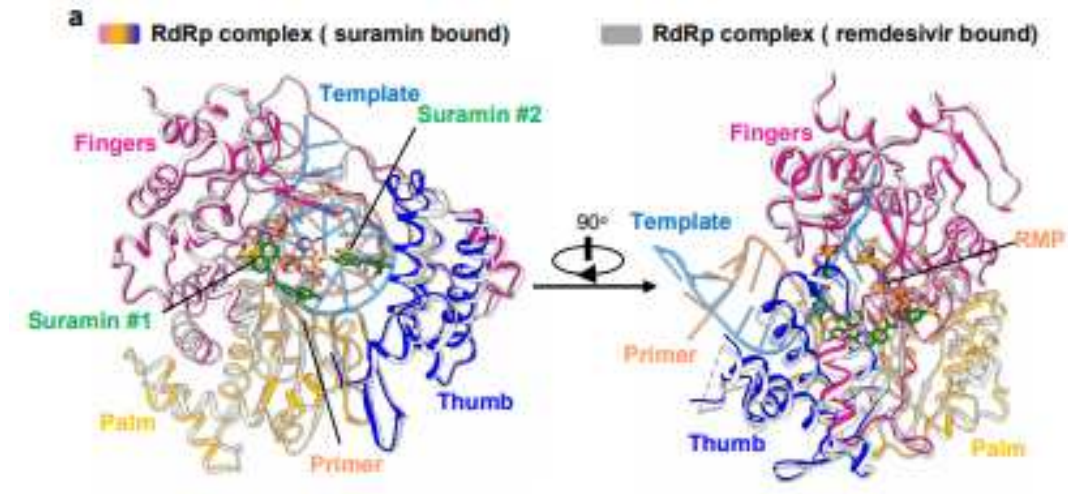

b
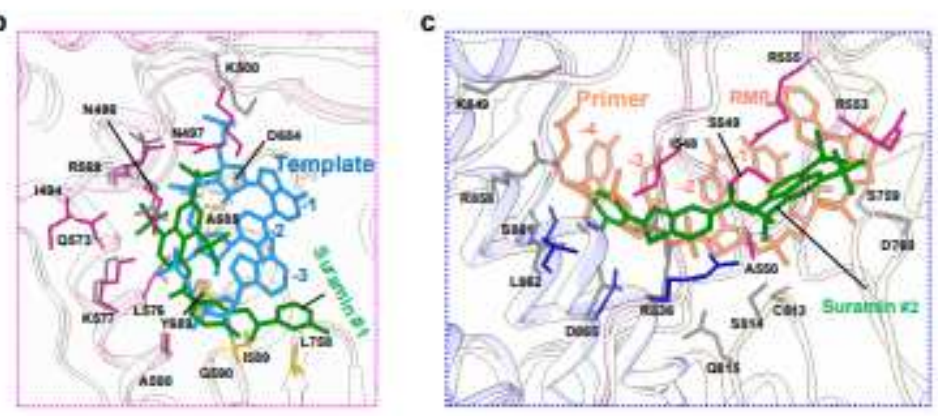

Figure 5

Inhibition mechanism from comparison with the remdesivir-bound RdRp structure a. The two overall views of the RdRp-suramin complex overlapped with the remdesivir-bound RdRp structure (PDB ID: 7BV2). For clarity, only the polymerase domains are presented. The remdesivir bound RdRp structure is set as light gray, the template RNA is set as cyan, and the primer RNA is set as red. b. Close view of suramin \#1 overlapped with RNA template strand. c. Close view of suramin \#2 overlapped with RNA primer strand. 

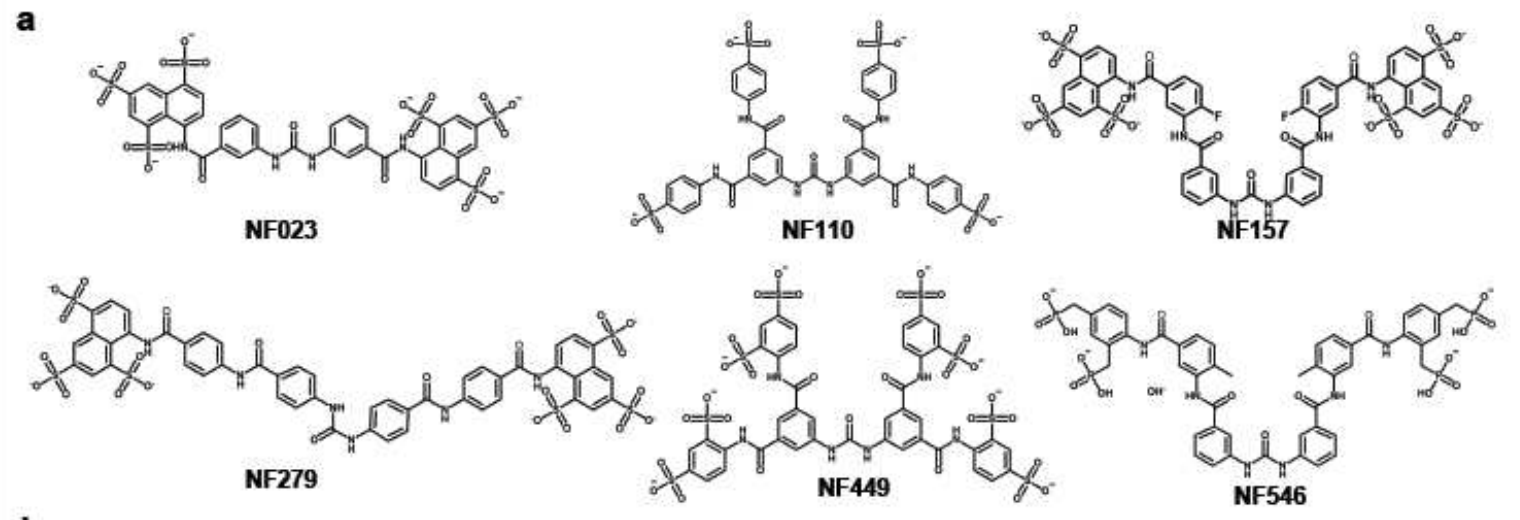

b
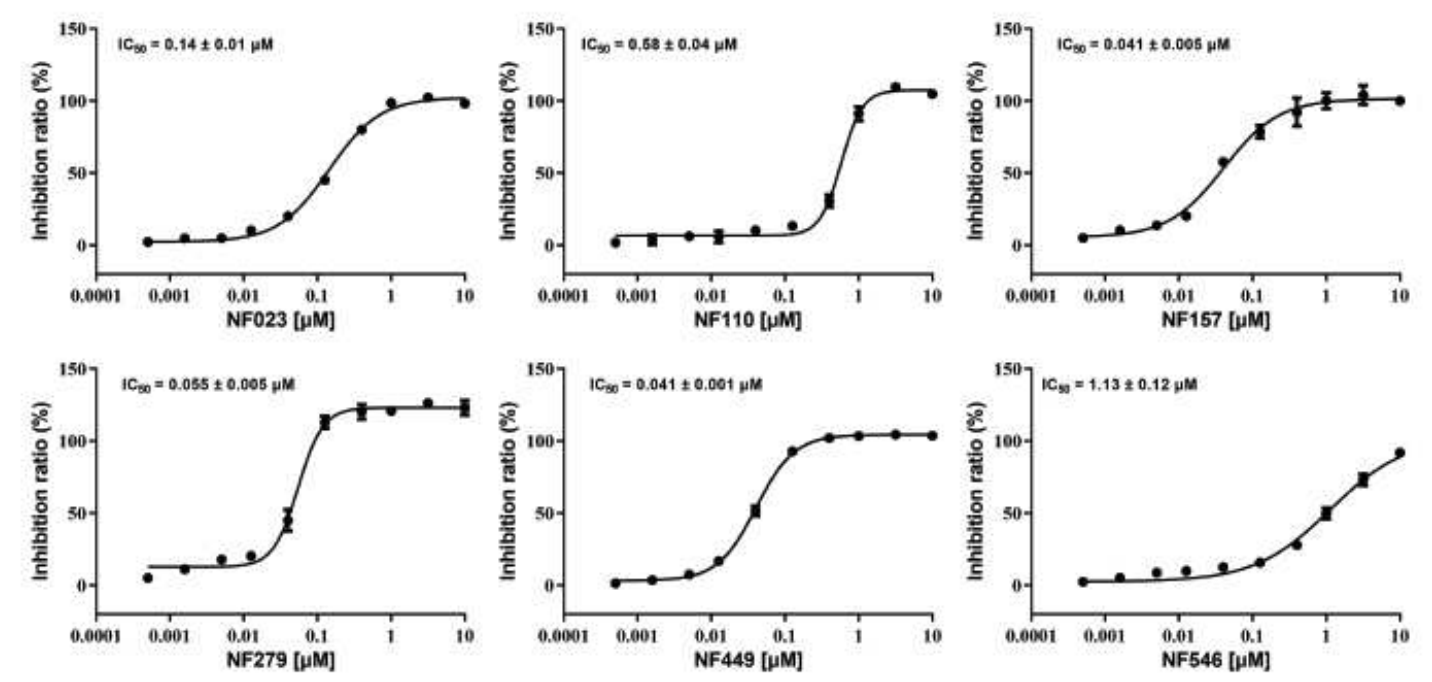

c
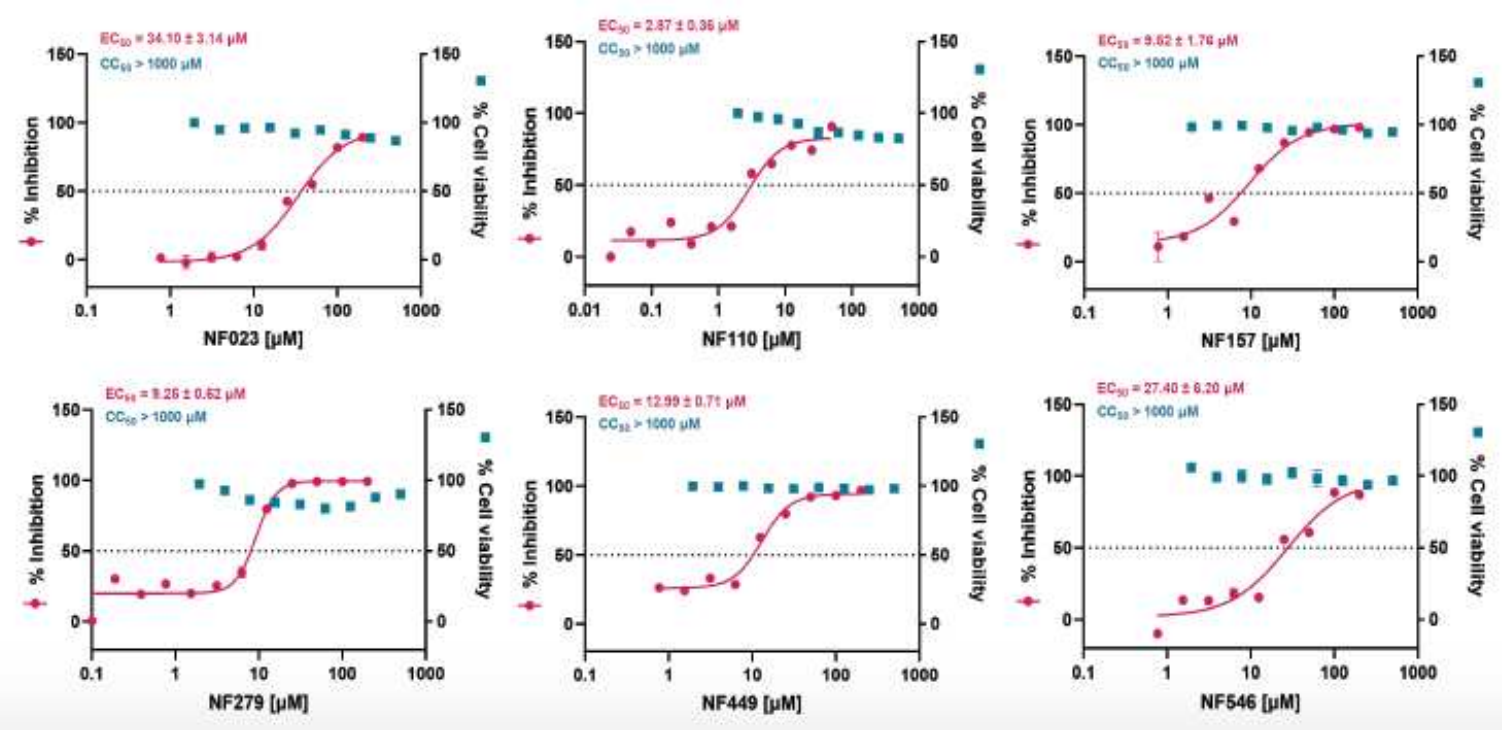

\section{Figure 6}

Inhibition SARS-CoV-2 RdRp by suramin derivatives. a. The chemical structures of suramin derivatives. b. The inhibition of RdRp activity by suramin derivatives. c. Inhibition of viral replication and cellular toxicity of suramin derivatives. 